\title{
Exotics Searches for New Physics \\ with the ATLAS Detector
}

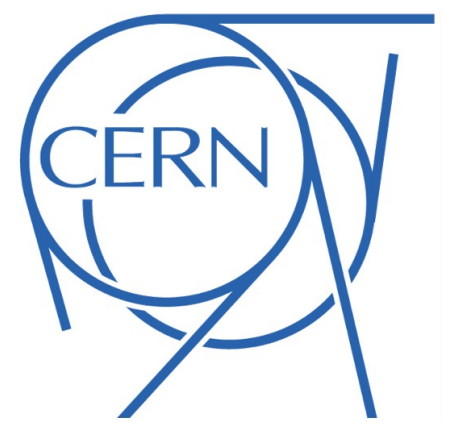

Jalal Abdallah

IFAE - Barcelona

on behalf of ATLAS Collaboration

19th Particles \& Nuclei International Conference

24-29 July 2011 


\section{Outline}

> The LHC has reached a new milestone with more than $1.3 \mathrm{fb}-1$ of deliver integrated luminosity

- As it is a "mission impossible" task to cover all the Exotics Searches in ATLAS in 15 min (over 19 CONF Notes and 11

Papers), I will give the headlines of the following analysis:

- Monojet + missing Et

- Dijet final states

- Dilepton final states

$>$ Lepton and missing Et

- Semileptonic ttbar

> Diphoton final states
Large diversity of theoretical models (Extra-Dimensions, Sequential SM, Lepto-Quark, ...) giving alternative solutions to the hierarchy problem, weakness of gravity dark matter, grand unification, etc.

- ATLAS Exotics public results web page 


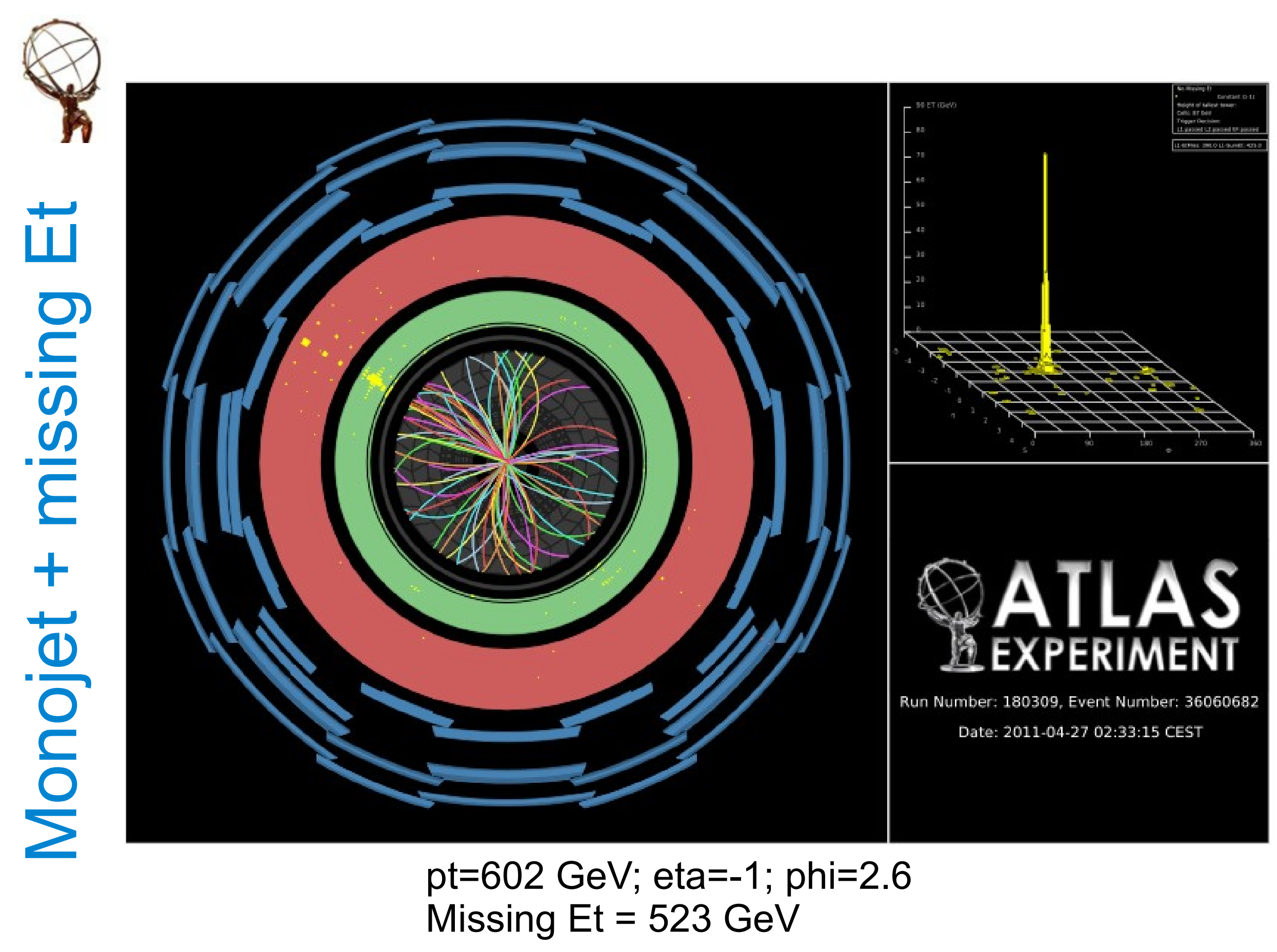




\section{final states}

Physics Model:

Arkani-Hamed,Dimopoulos,Dvali

(ADD) Kaluza-Klein Graviton

- Observable

> Large missing Et

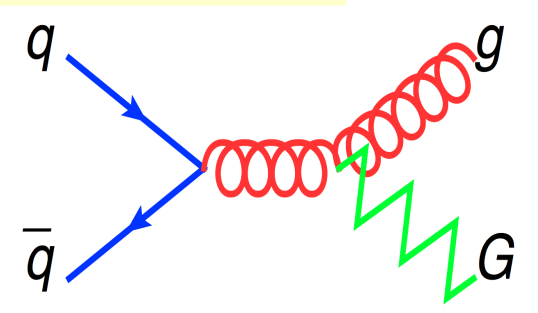

> High pt jet

> Event Selection

> High pt jet and large MET

> No second jet above threshold

> No reconstructed lepton $(e, \mu)$

> Main background

> EW SM (Z/W+jets)

PANIC- July 28th, 2011
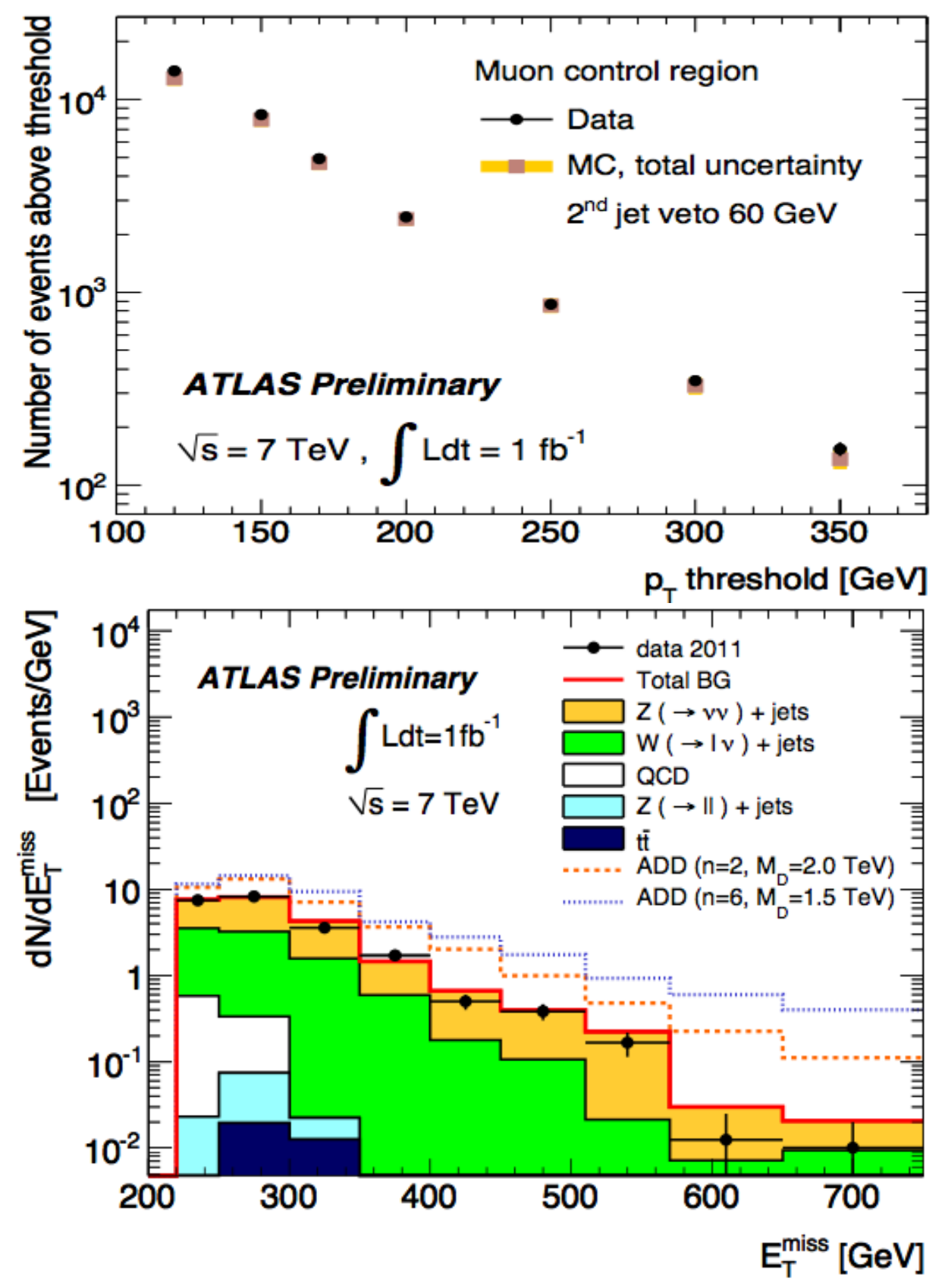


\section{h \\ Monojet+MET final states}
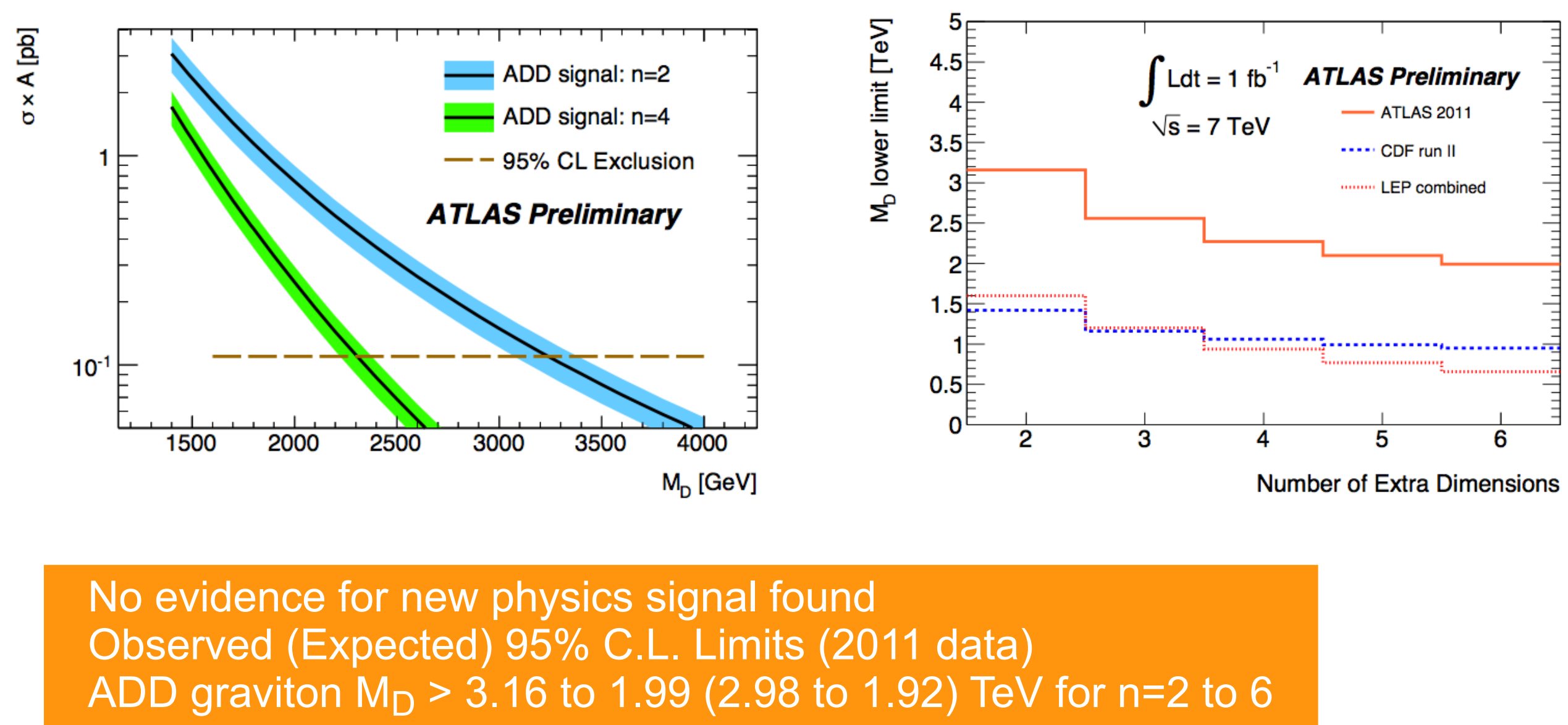


\section{ATLAS-CONF-2011-095 \\ Dijet final states: resonances}

Physics Models:

Excited quarks q*

Axigluons

Color-octet scalars

- Observable

> Dijet invariant mass

> Events Selection

> Two highest pt jets with $|\eta|<2.5$ and $\left|\Delta \eta_{j j}\right|<1.3$

> Background

- SM QCD parametrization $f(x)=p_{1}(1-x)^{p_{2}} x^{p_{3}+p_{4} \ln x}$

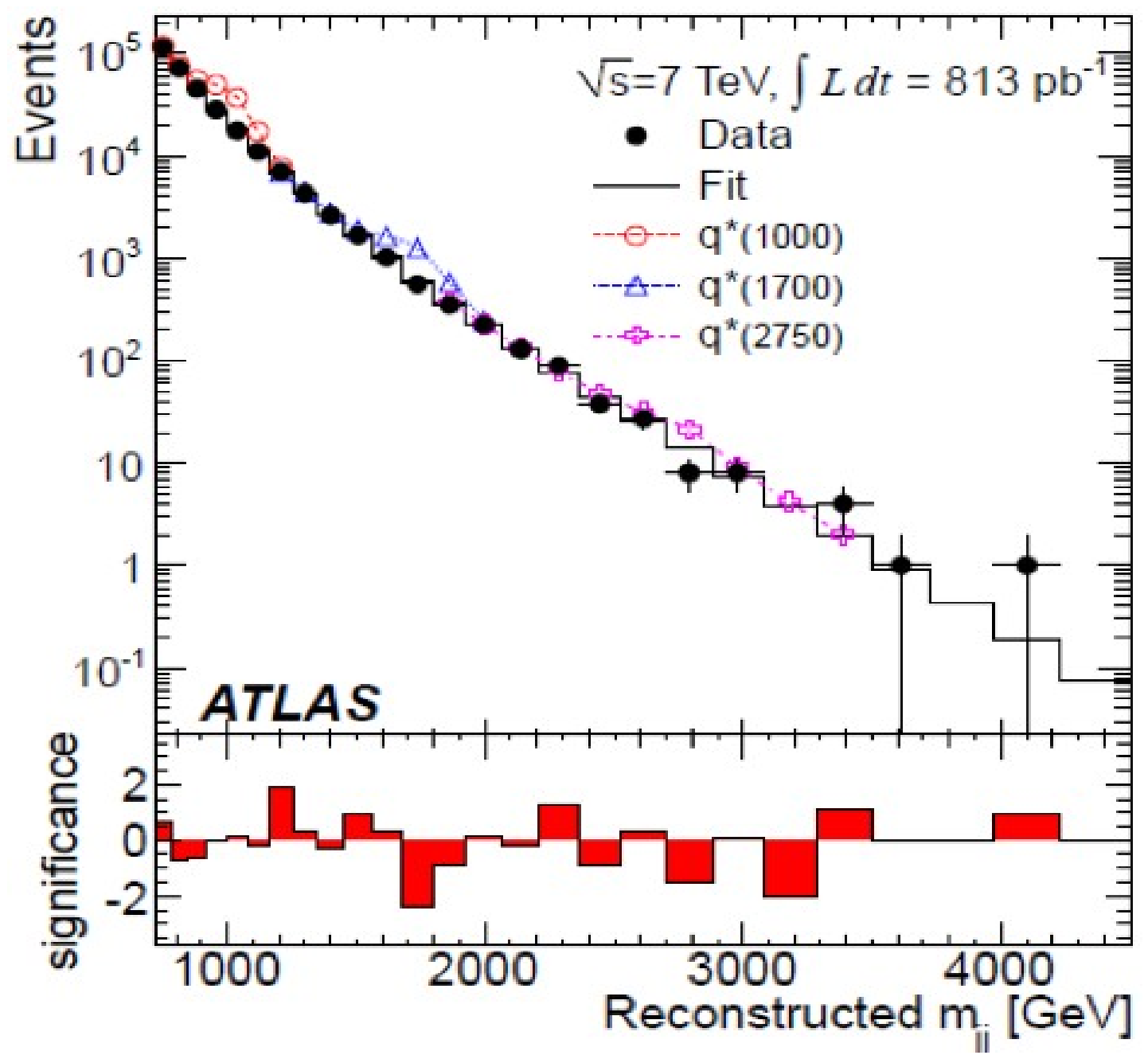




\section{Dijet final states:}

Physics Model:

Quark contact interactions

Randall-Meade QBH

$\chi$ : Centrality in $\mathrm{cm}$ frame

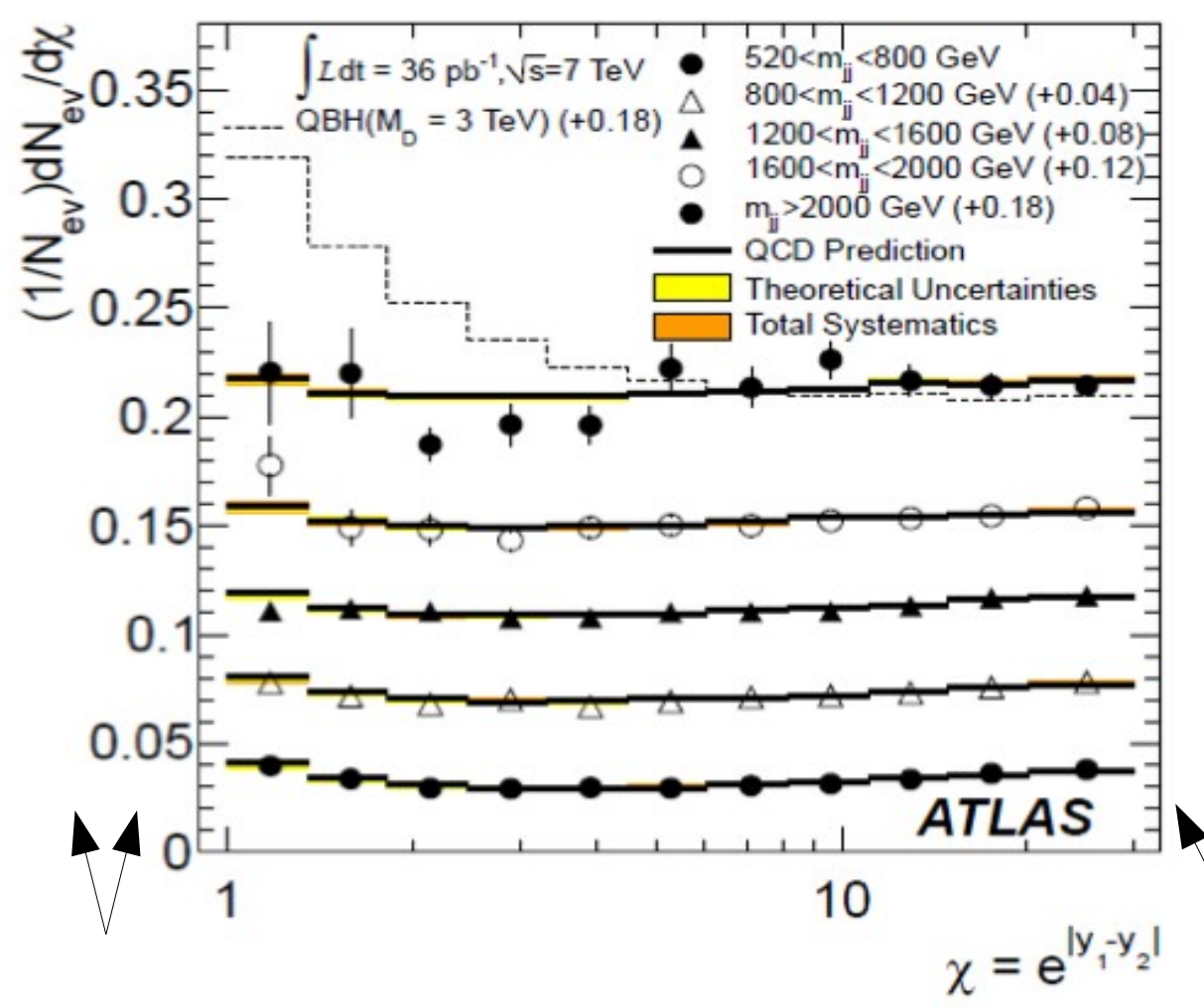

> Observable

> Background

- Dijets angular distribution

> Event Selection

$F_{\chi}$ : Centrality ratio in $\mathrm{cm}$ frame

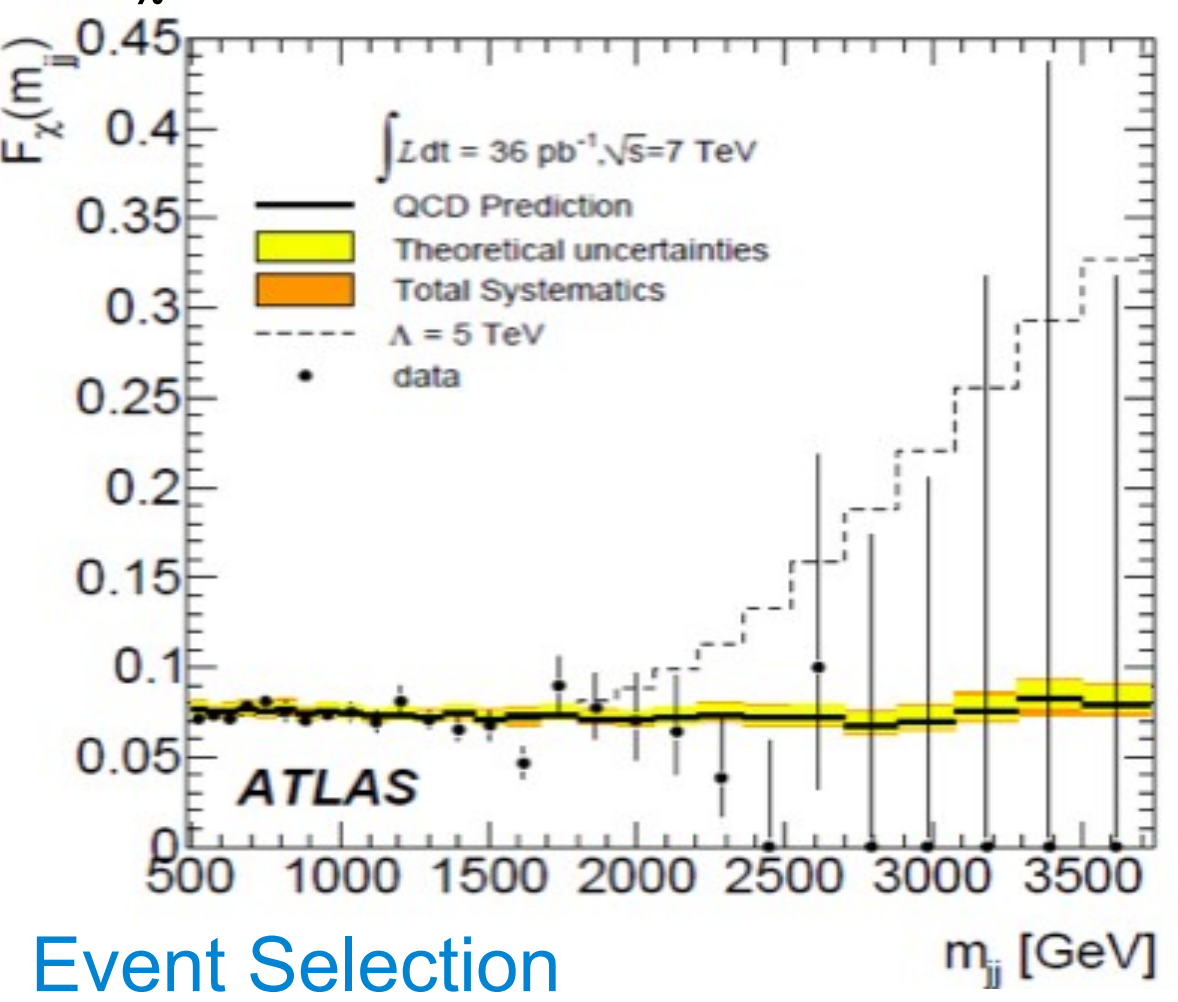

- At least two jets with

$p t_{j 1}>60 \mathrm{GeV}$ and $\mathrm{pt}_{\mathrm{j} 2}>30 \mathrm{GeV}$

> SM QCD (NLO) prediction 


\section{Dijet final states: Limits}

\section{No evidence for new physics signal found}

Observed (Expected) 95\% C.L. Limits (2011 data)

Excited quarks $\left(\mathrm{q}^{*}\right) \quad \mathrm{M}>2.77$ (2.91) $\mathrm{TeV}$ Axigluons Color Octet Scalar $\quad M>1.71$ (1.91)

Observed (Expected) 95\% C.L. Limits (2010 data)

RMQ Black Hole $(n=6) \quad M>3.67$ (3.64) TeV Contact interaction $\Lambda>6.7(5.7) \mathrm{TeV}$

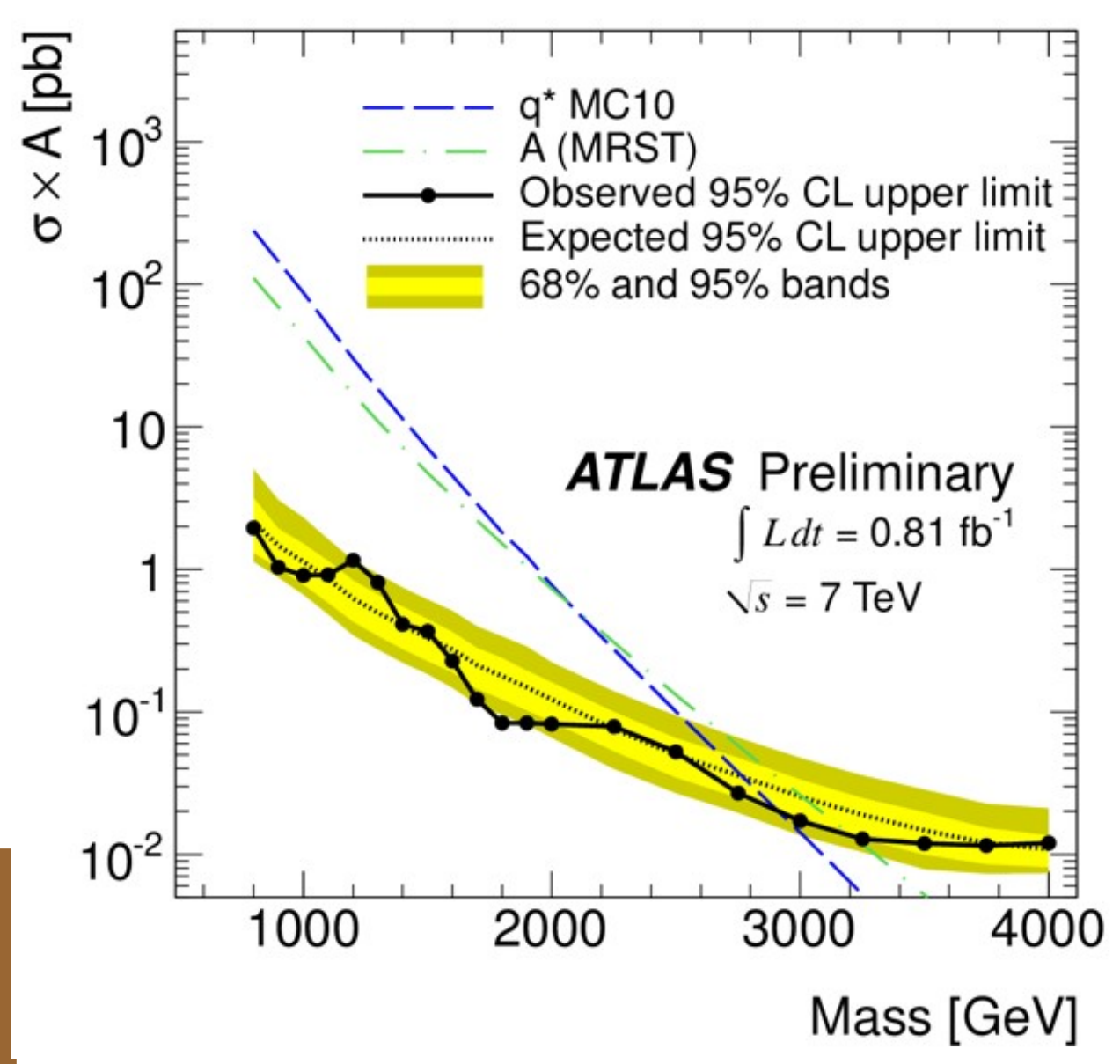




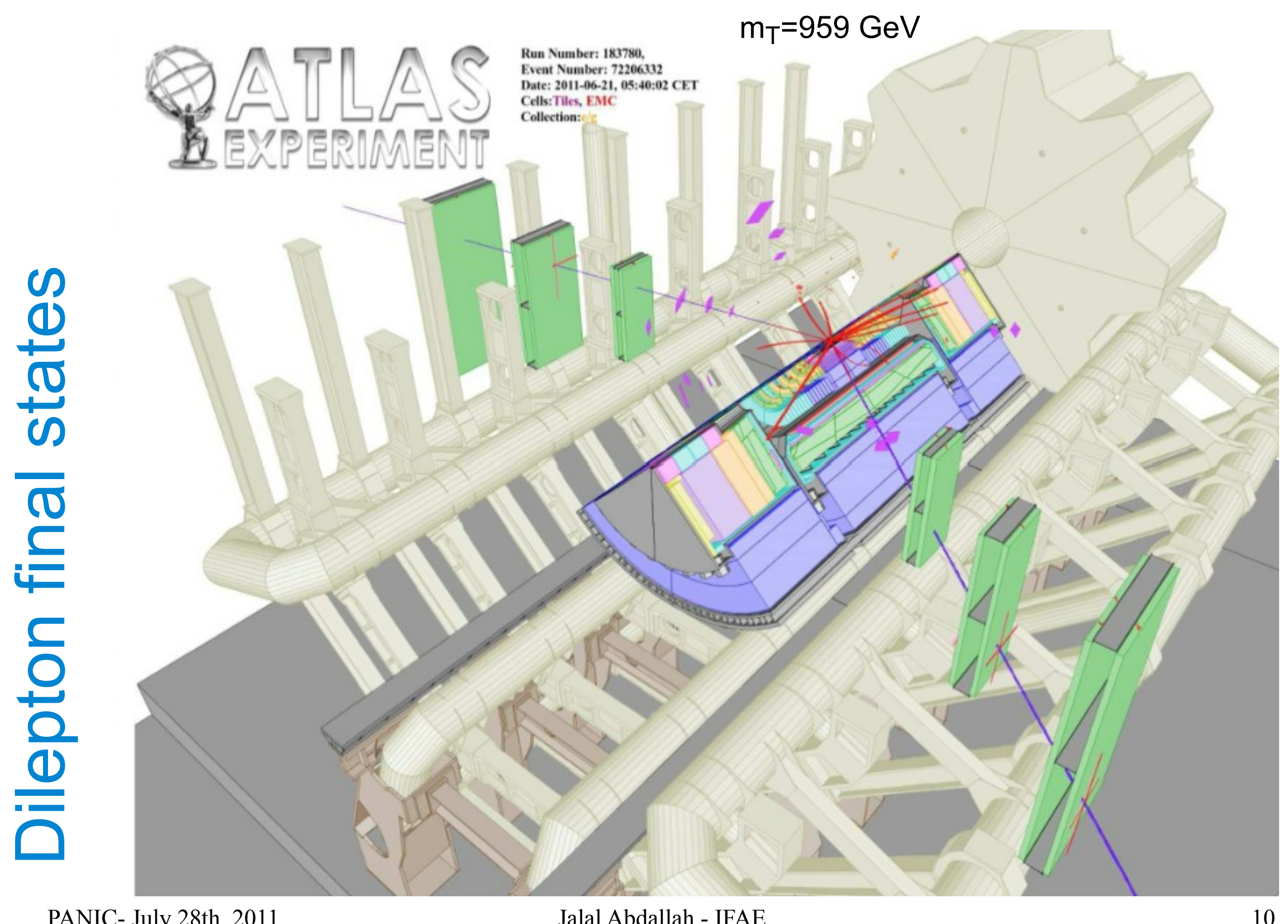




\section{Dilepton final states}

ATLAS-CONF-2011-083

Physics Models:

Z' (SSM, E6)

Randall-Sundrum Graviton

Contact interactions

- Observable

> Dilepton invariant mass

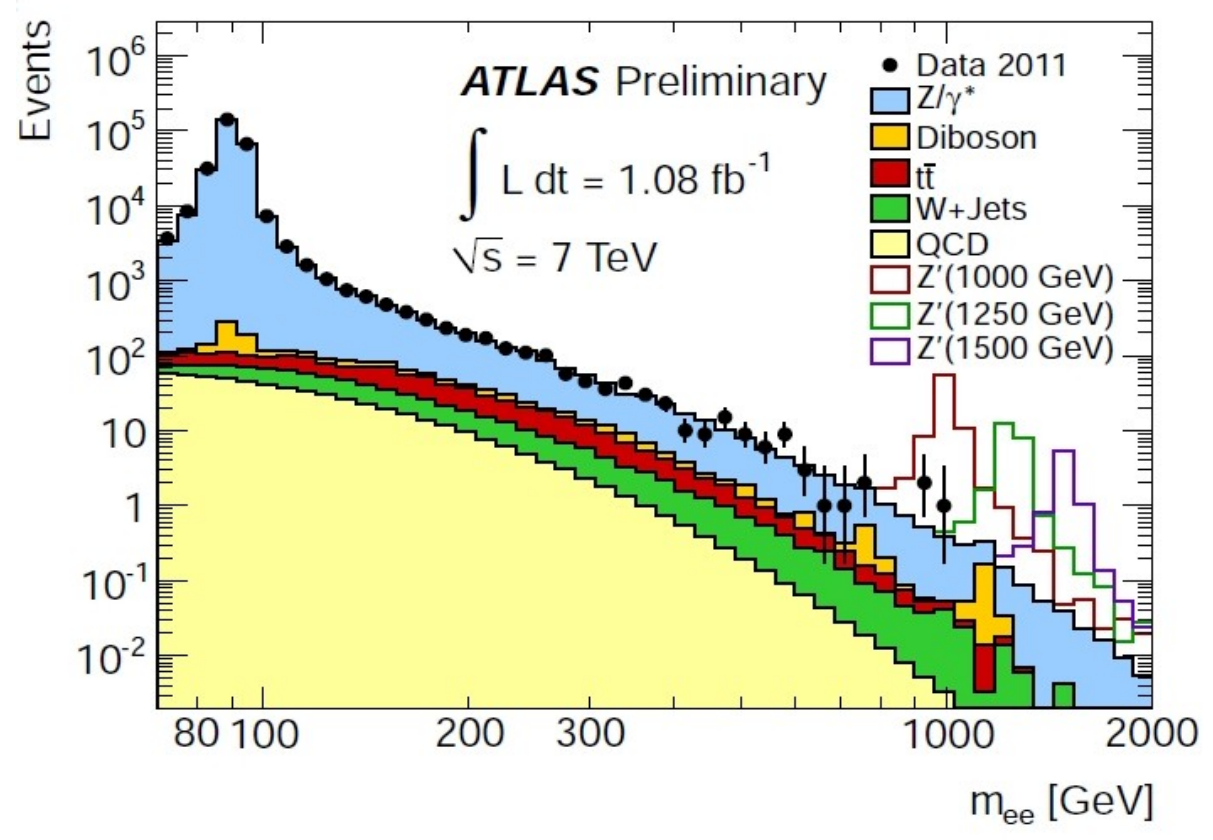

> Event Selection

- Select events with two leptons (same flavor, e or $\mu$ )

- Main background

- SM EW background $\left(\mathrm{Z} / \gamma^{*} \rightarrow \mathrm{II}\right)$

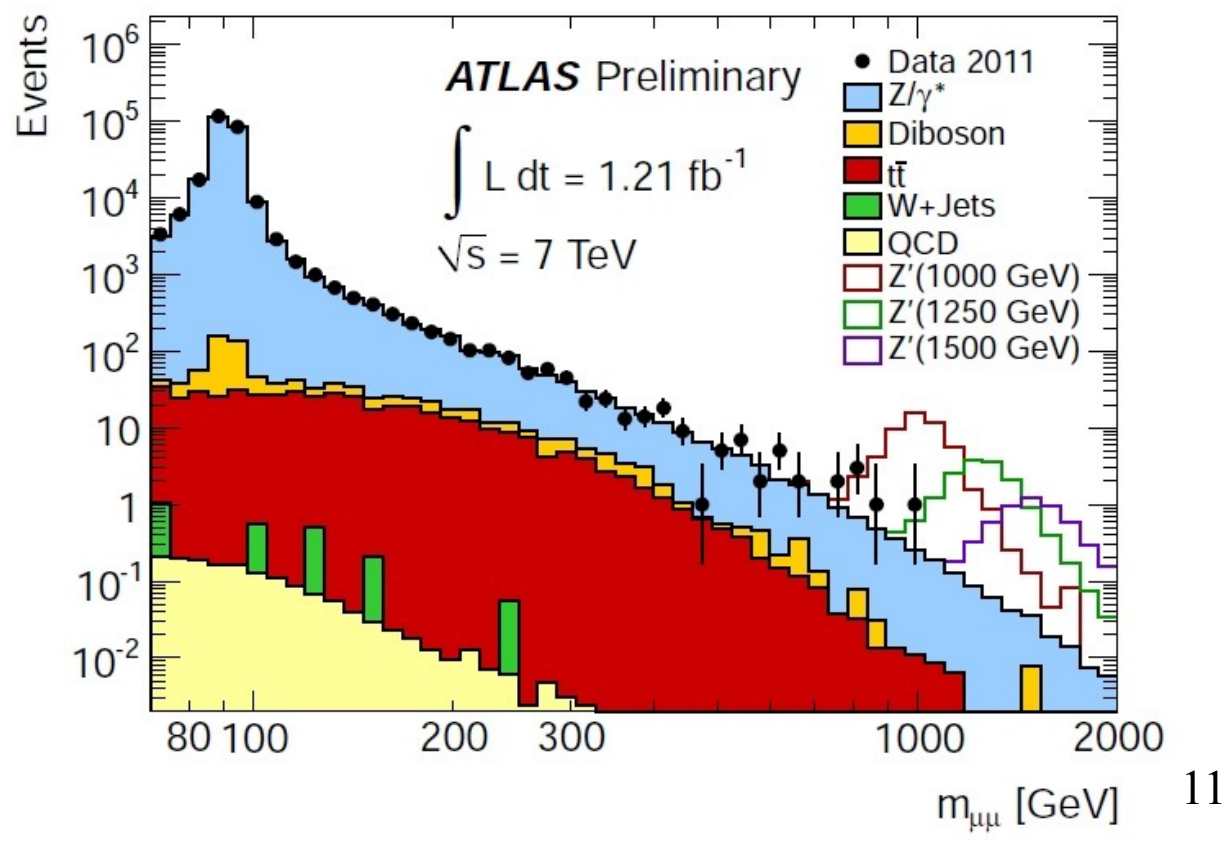




\section{Dilepton final states}
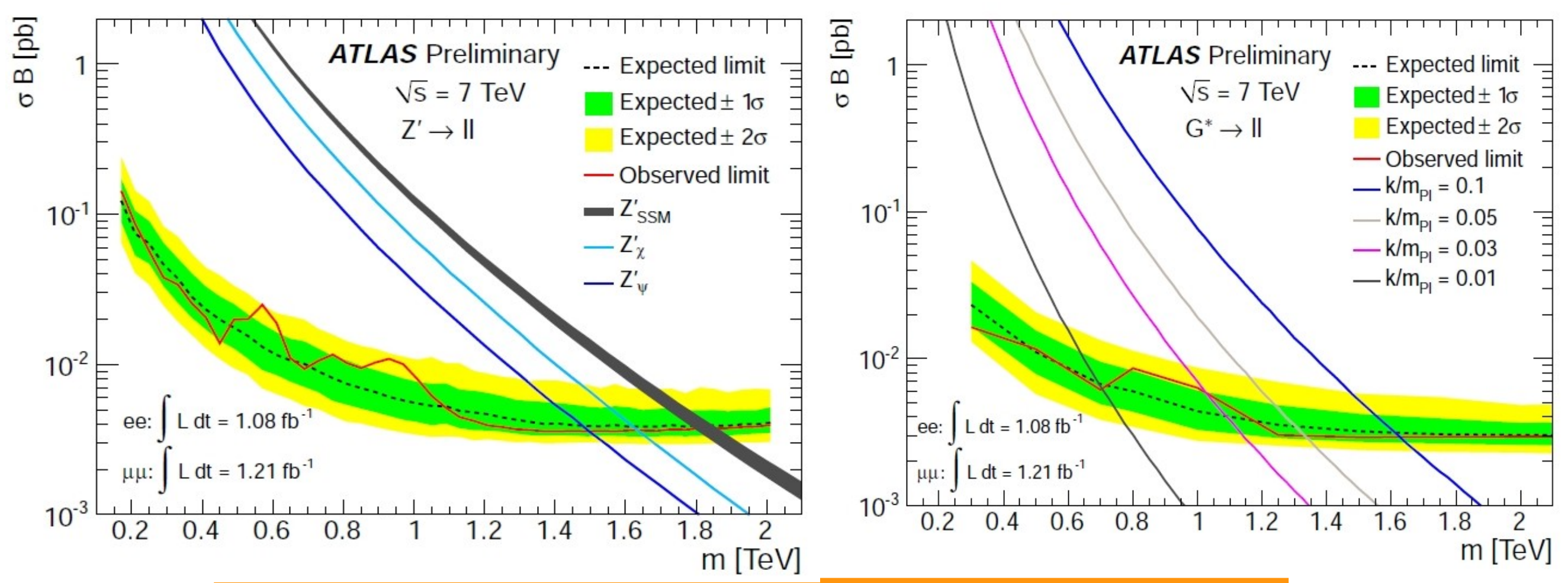

No evidence for new physics signal found

Observed (Expected) 95\% C.L. Limits (2011 data)

E6 Z' bosons $\mathrm{M}$ in the range 1.50-1.64 (1.50-1.64) TeV

Z' SSM

RS G, k/M=0.1

$M>1.83(1.83) \mathrm{TeV}$

RS G, k/M=0.01

$M>1.63(1.61) \mathrm{TeV}$

(2010 data) Contact interaction Dimuon $\wedge>4.9$ (5.1) TeV 


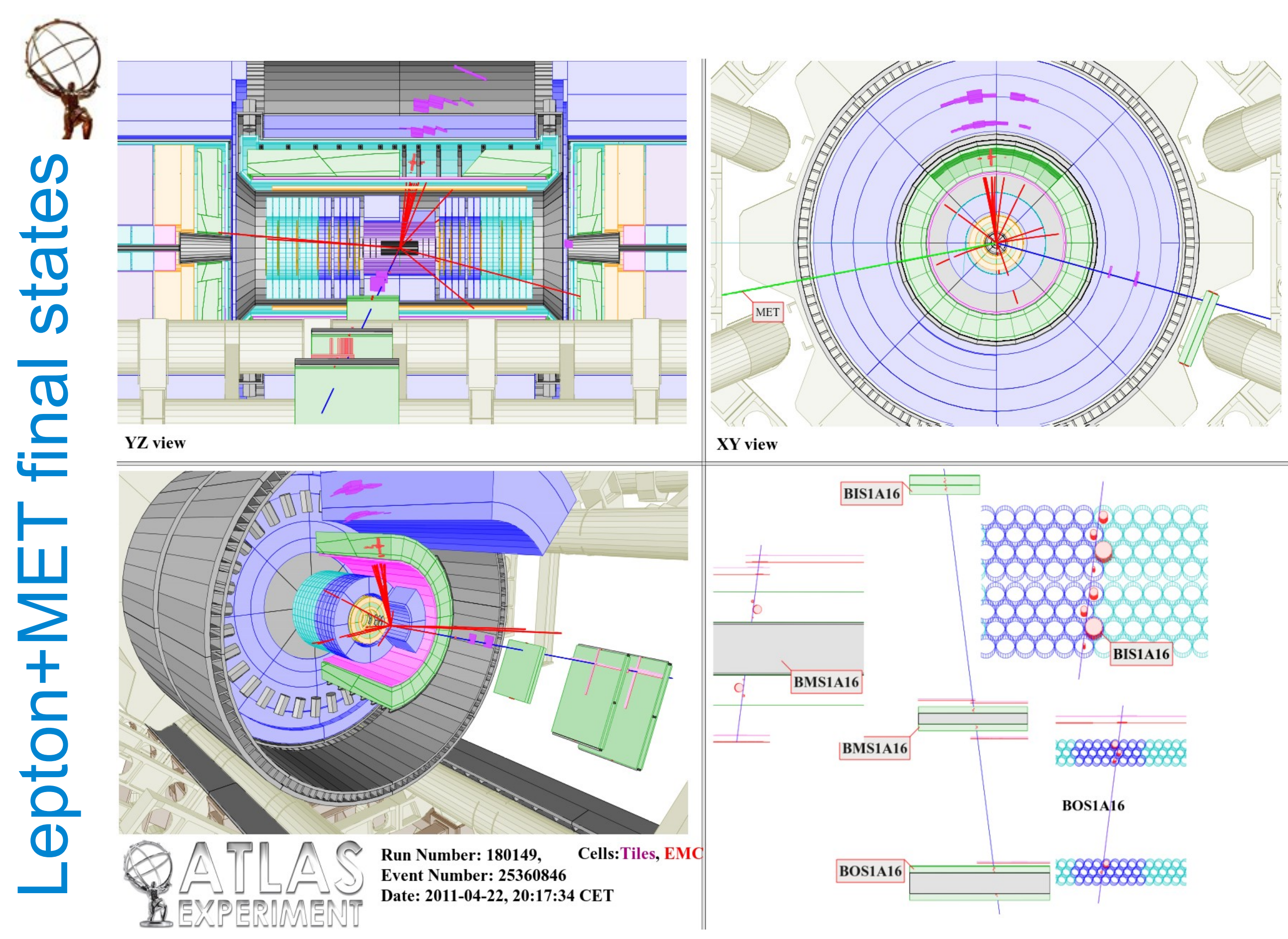

PANIC- July 28th, 2011

Jalal Abdallah - IFAE 


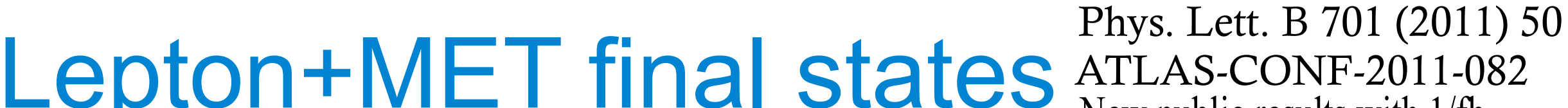

Physics Models:

W' SSM

$W^{*}$ Chiral boson

- Observable

> Transverse mass

> Event Selection

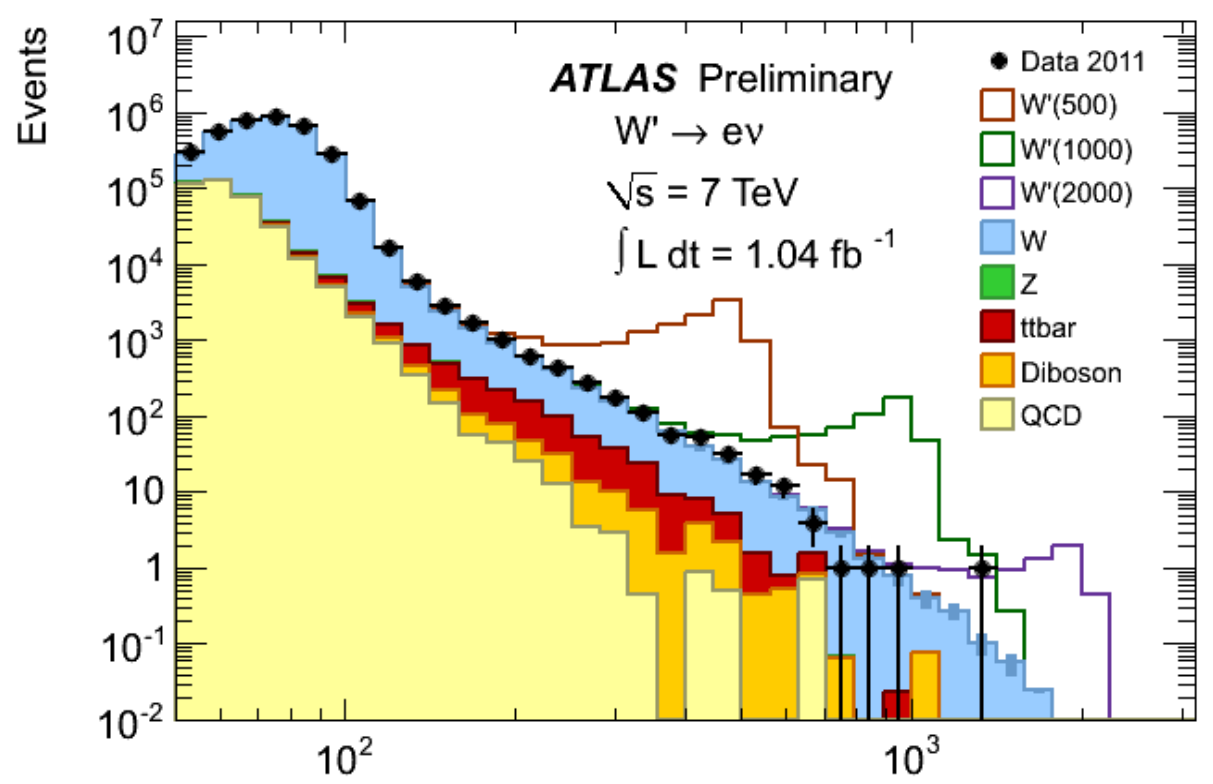

> Select events with just one high pt lepton $(e, \mu)$ and high MET

> Background

> SM EW background (W)

- QCD faking electron

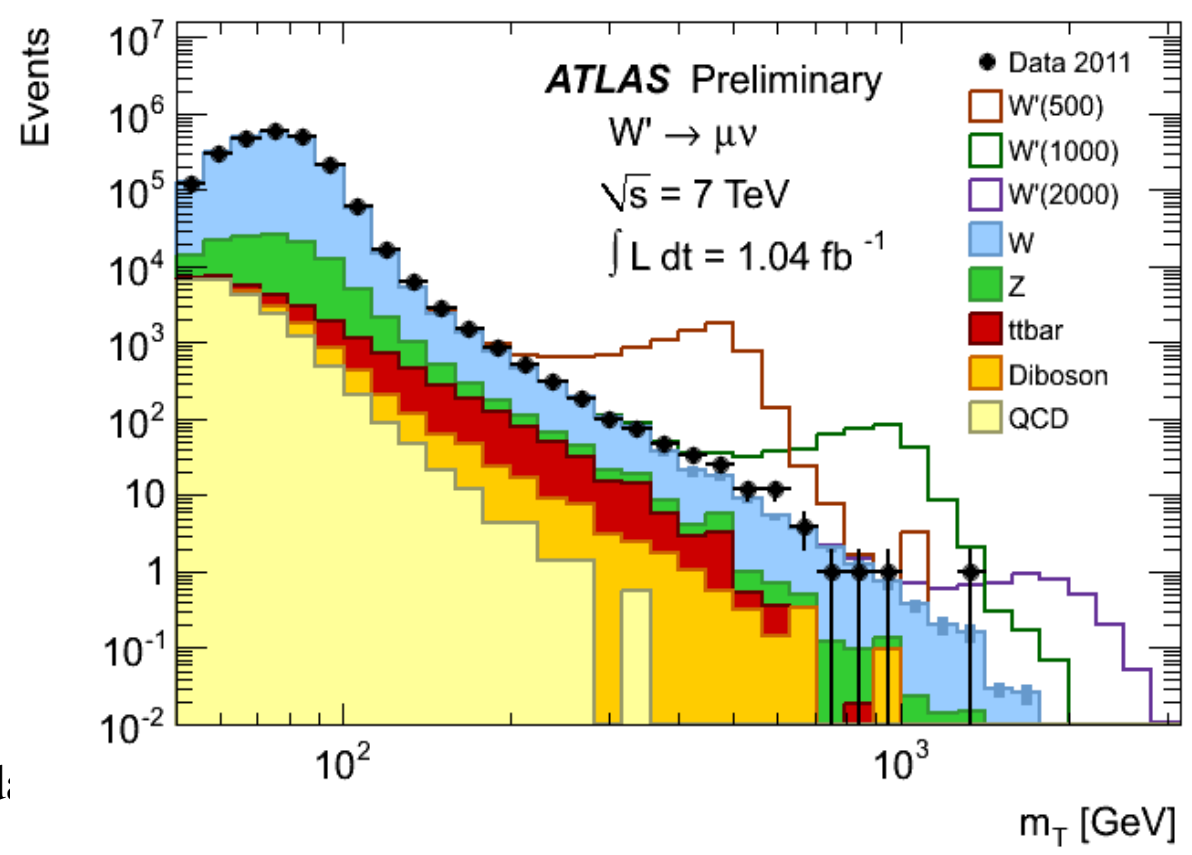




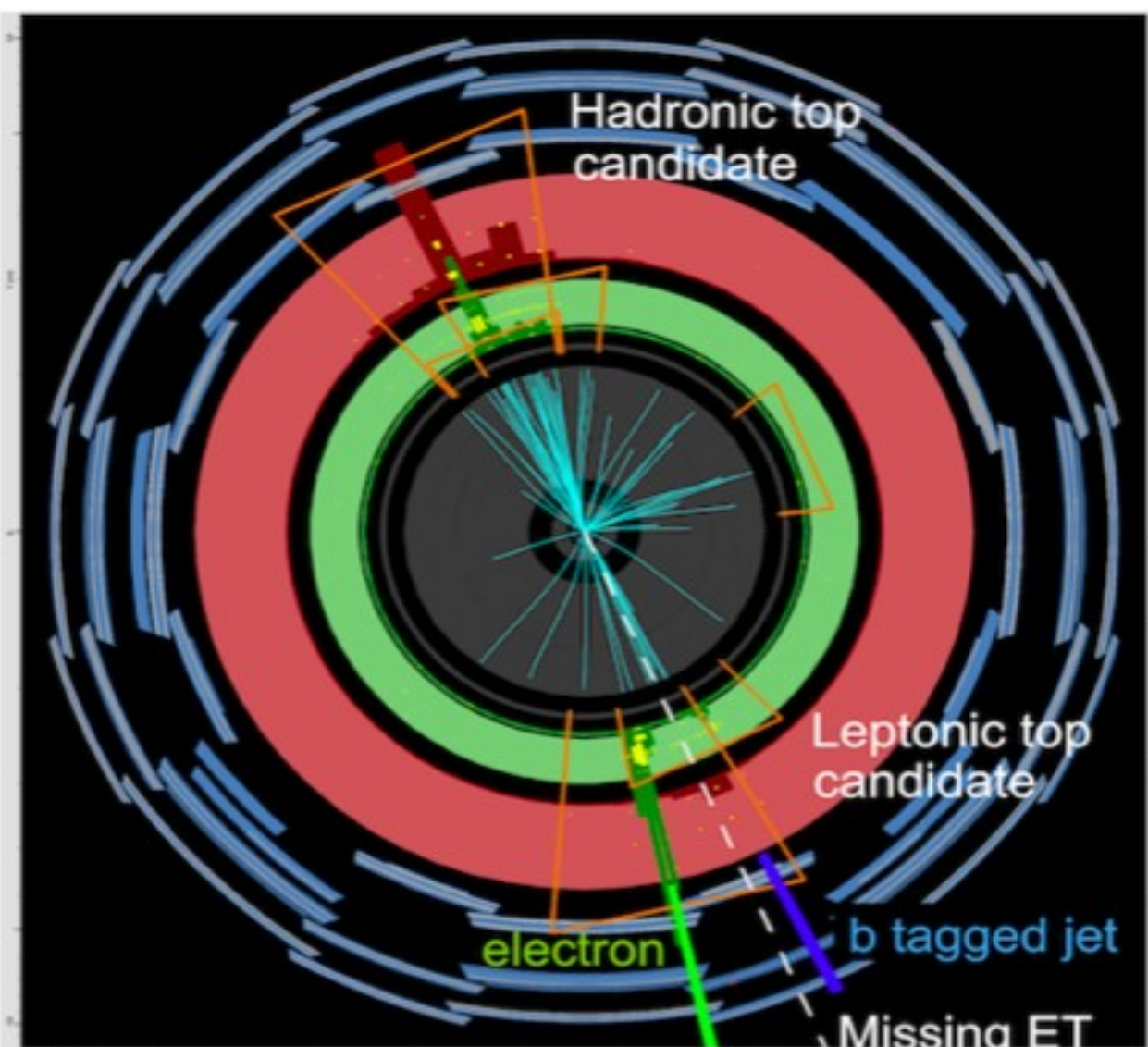

ATLAS 道EXPERIMENT

Run Number: 180400, Event Number: 54251178

Date: 2011-04-28 03:33:58 CEST

$$
\mathrm{m}_{\mathrm{tt}}=1602 \mathrm{GeV}
$$

\section{Leptonic top candidate of \\ cy}

Hadronic top candidate

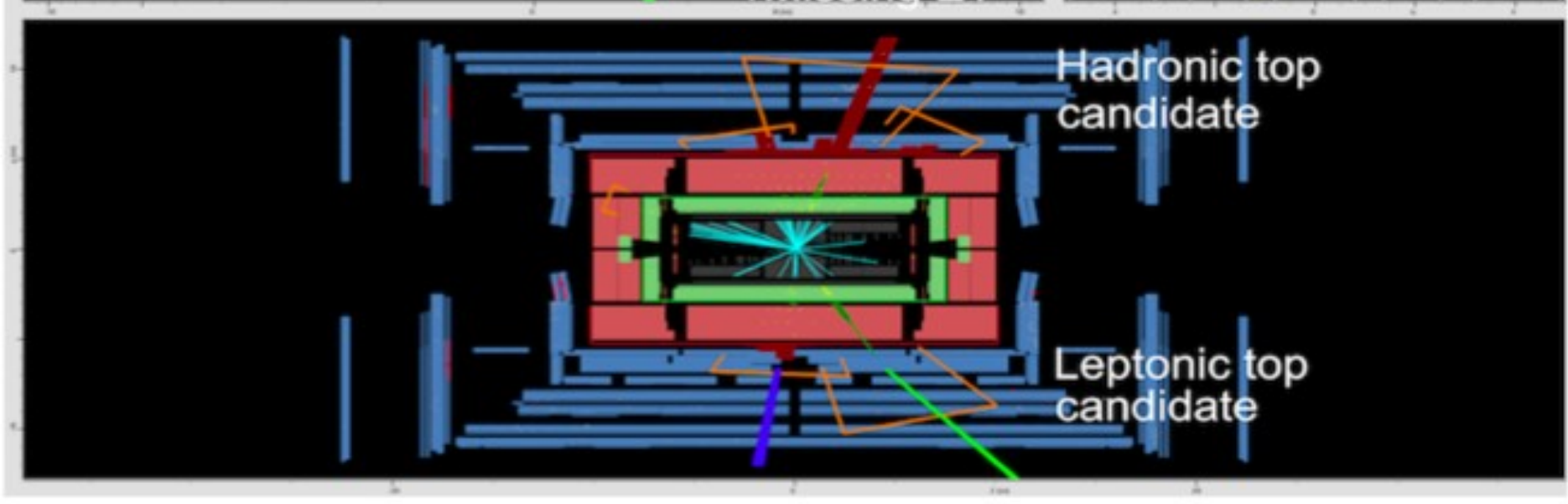

PANIC- July 28th, 2011

Jalal Abdallah - IFAE 


\section{Semileptonic ttbar final states}

Search for ttbar resonances:

Kaluza-Klein gluon

Z' (TC2 leptophobic)

> Observable

- Invariant mass of the jets, lepton and MET

> Selection

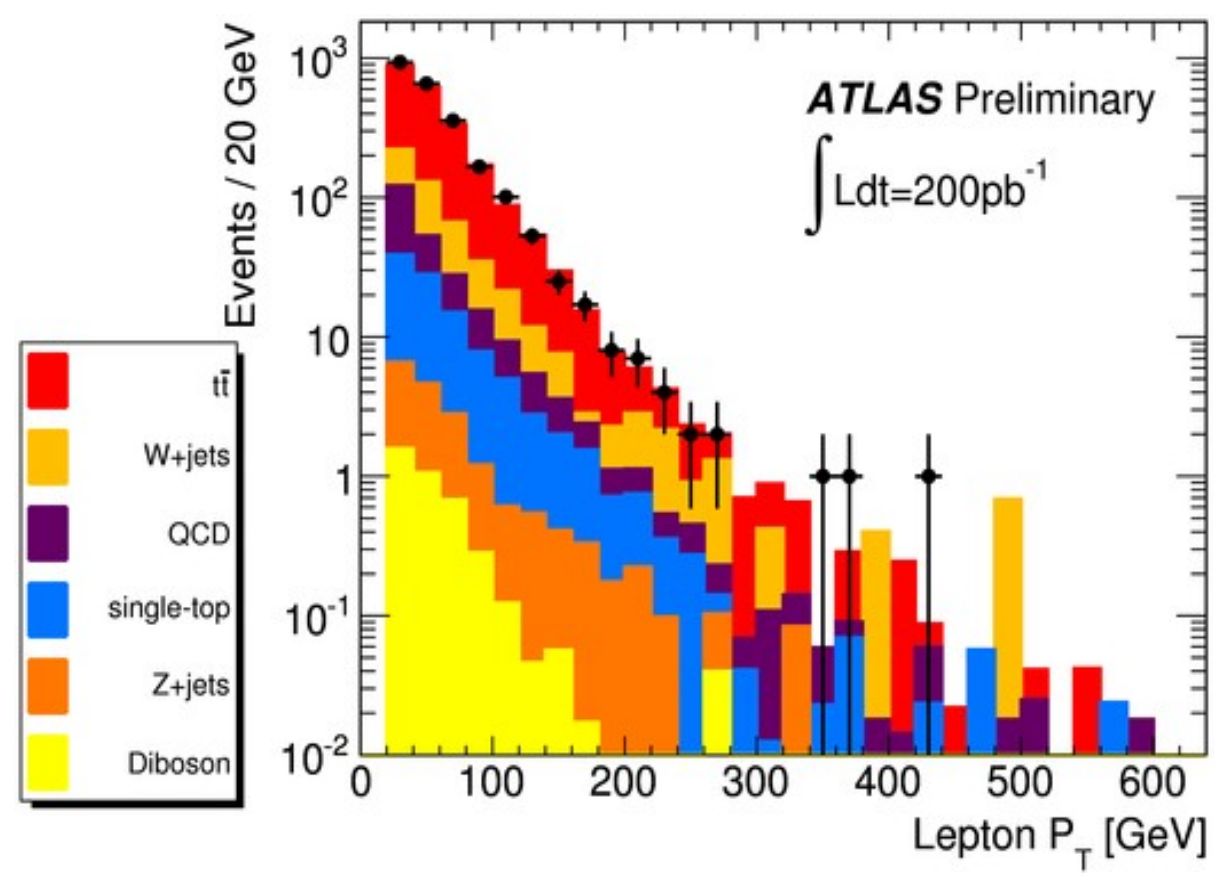

$>=4$ jets

$>>=1 \mathrm{~b}-$ jet

> Just one lepton, pt > $20 \mathrm{GeV}$

> Large MET

- Background

- Main background SM ttbar

PANIC- July 28th, 2011

Jalal Abd:

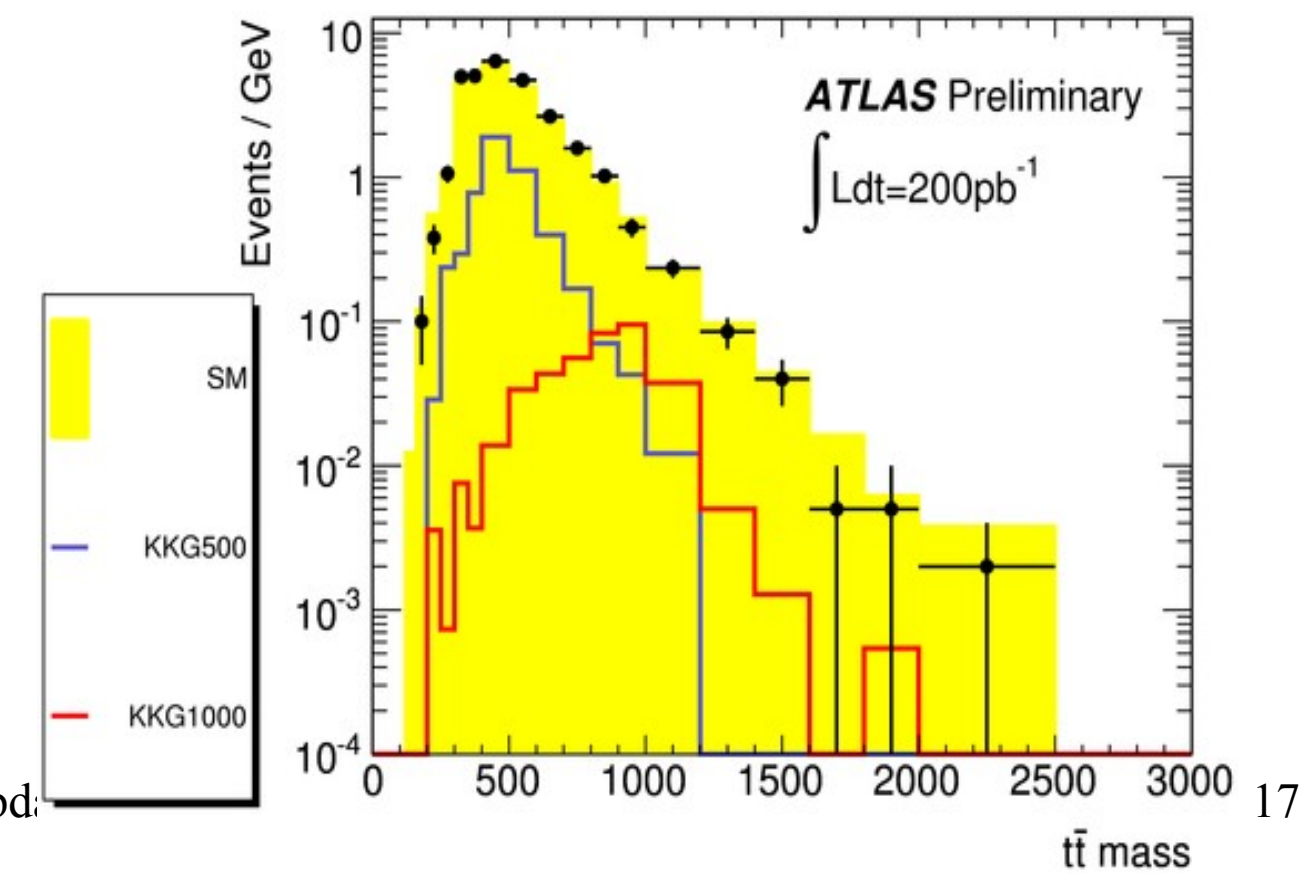




\section{ATLAS-CONF-2011-087}

\section{Semileptonic ttbar final states}
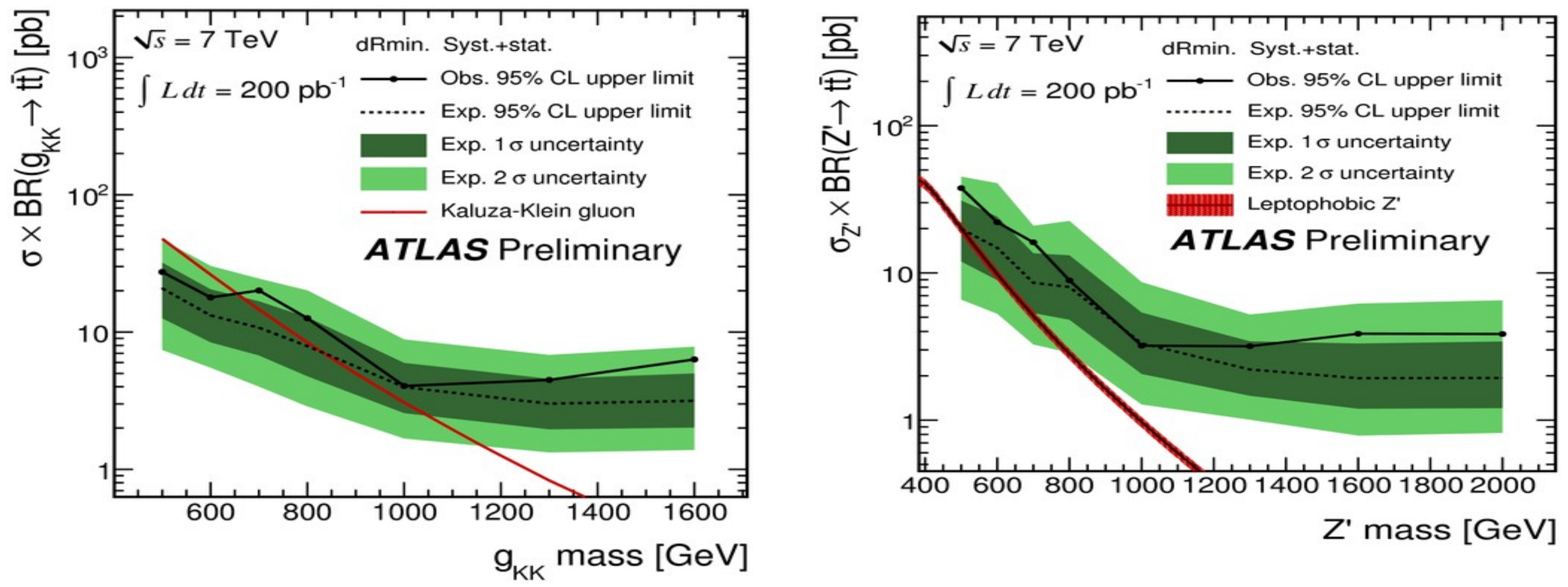

No evidence for new physics signal found

Observed (Expected) 95\% C.L. Limits

(2011 data)

KK-gluon M>650 (825) GeV

No exclusion limits on Z' TC2 Leptophobic 


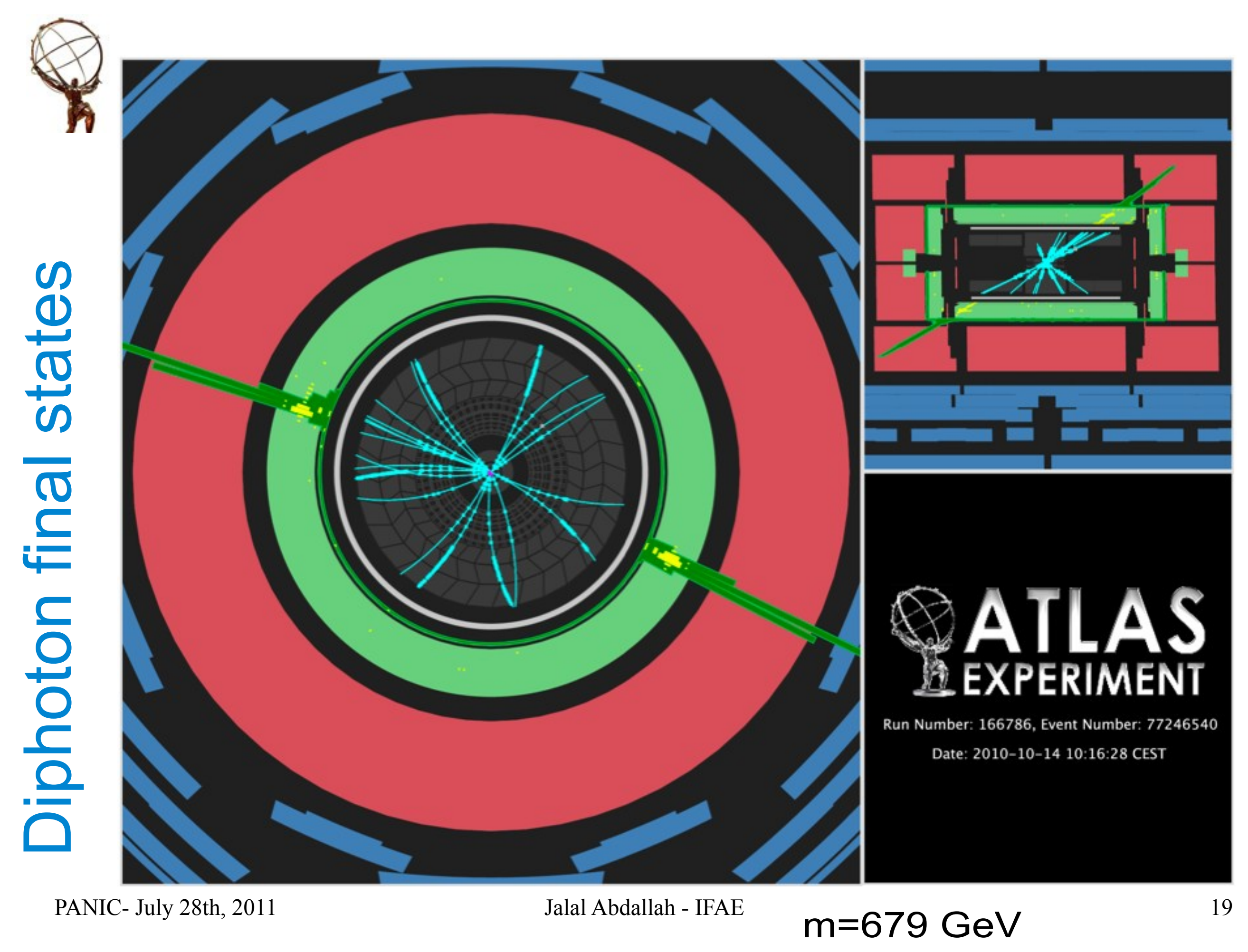




\section{Diphoton final states}

Physics Model: Randall-Sundrum Graviton
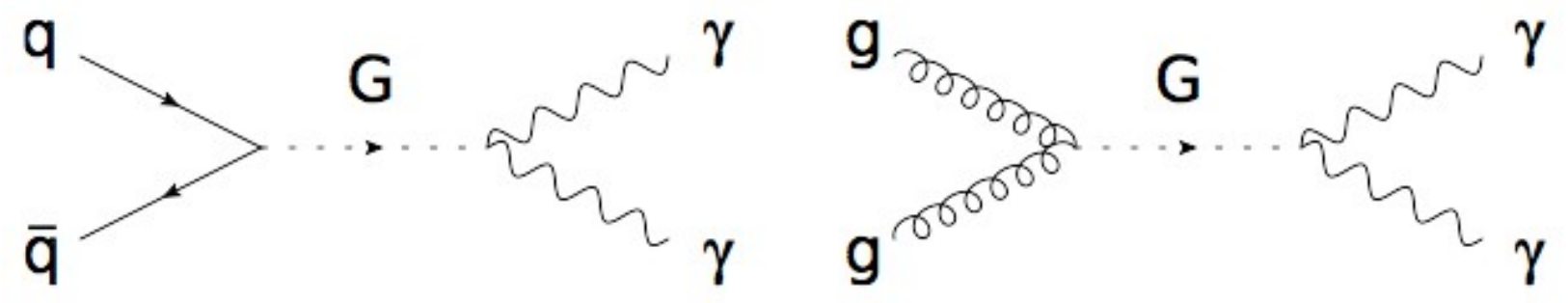

> Observable

> Diphoton invariant mass

> Selection

> Two photons with pT>25 GeV

> Background

- SM diphoton production

> fake photons from QCD

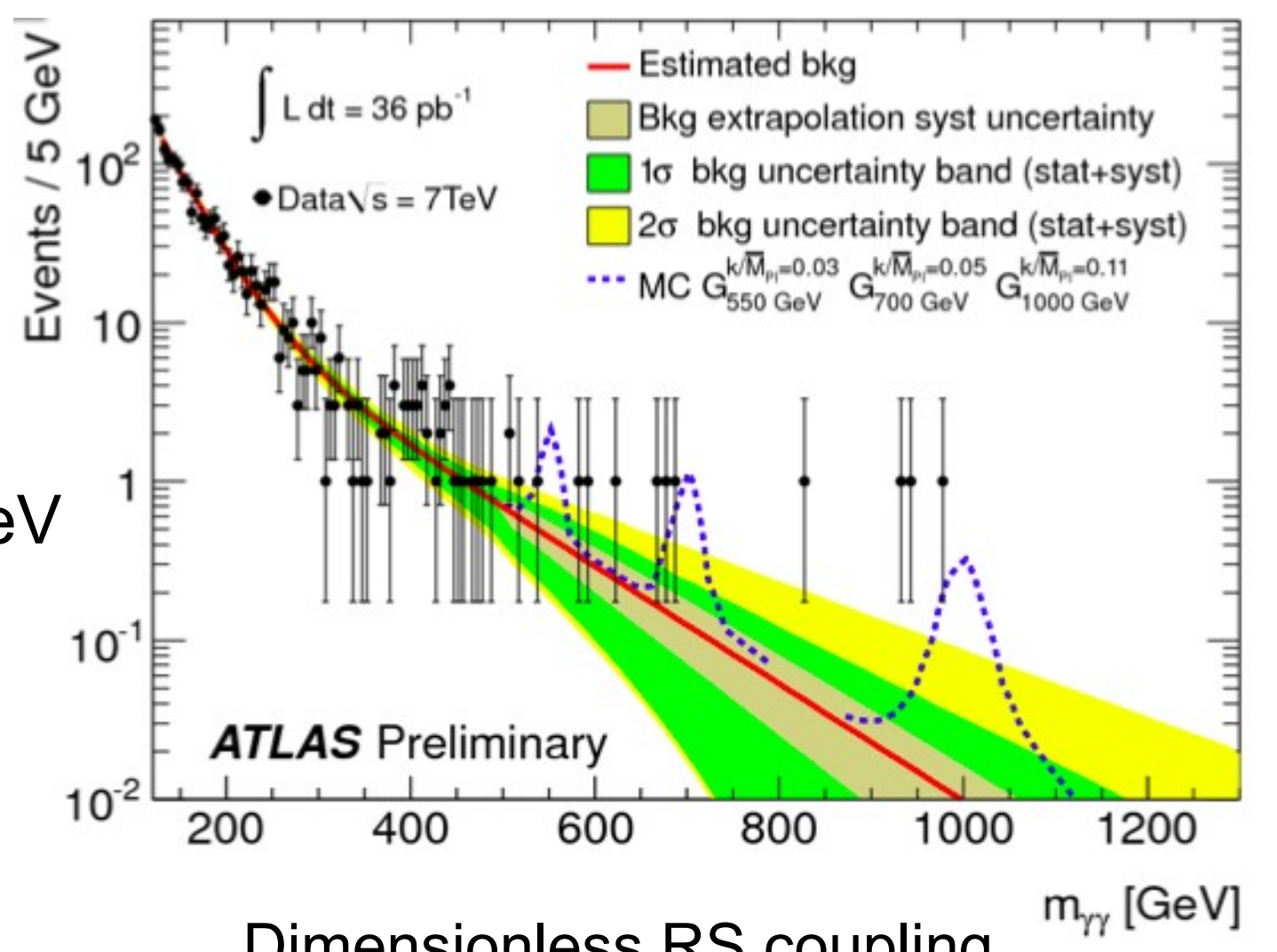

Dimensionless RS coupling $\mathrm{k} / \mathrm{M}_{\mathrm{pl}}$ (from 0.02 to 0.1 ) 


\section{Diphoton final states}

ATLAS-CONF-2011-044
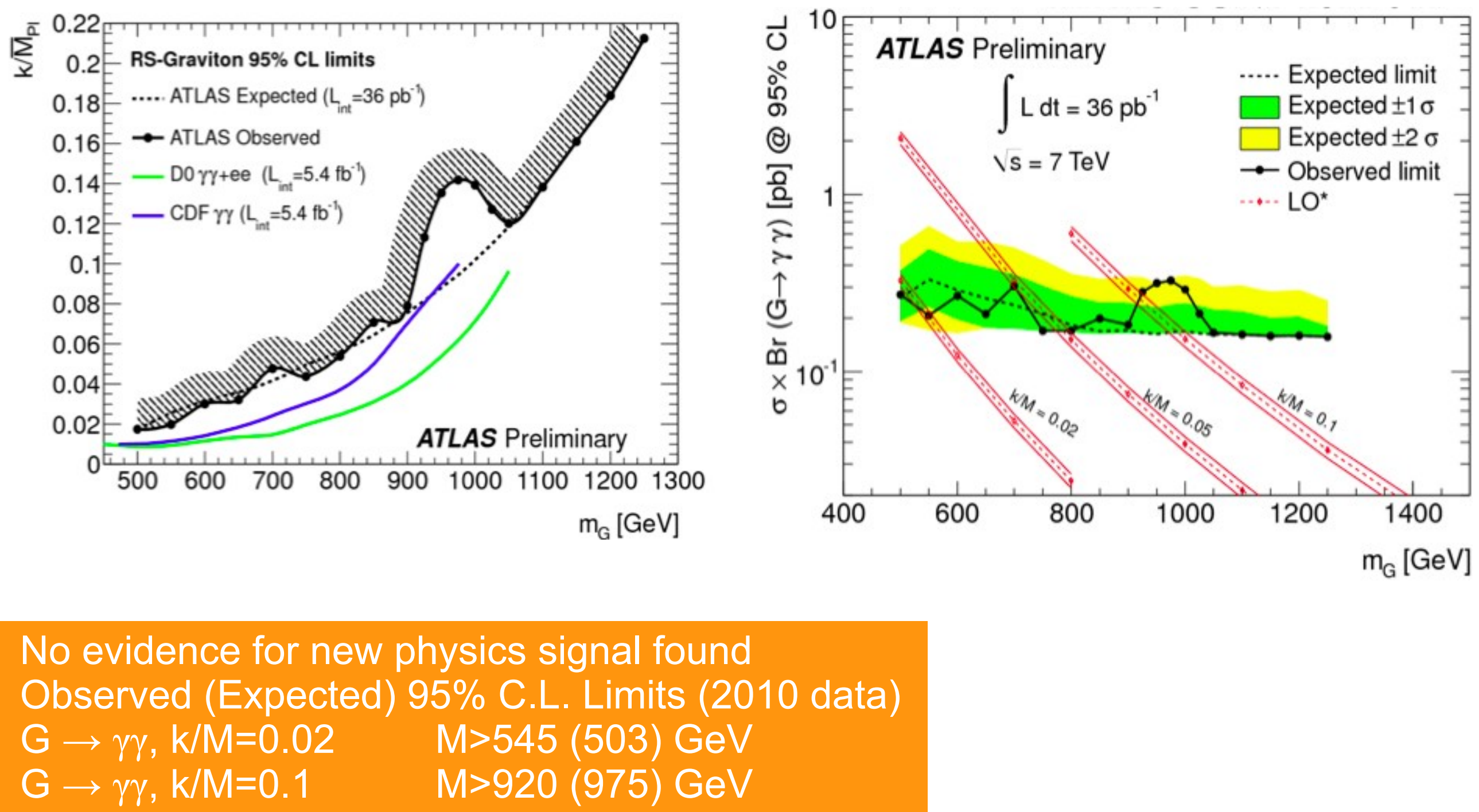

PANIC- July 28th, 2011

Jalal Abdallah - IFAE 


\section{Summary}

> Very good performance for the LHC in 2011.

- ATLAS data taking has been very efficient, recording up to $1.3 \mathrm{fb}^{-1}$.

- ATLAS Exotics searches for New Physics showed no significant excess beyond the Standard Model predictions

- Most of the observed limits are already beyond Tevatron results, and some of them are world best records 


\section{ATLAS Searches* - 95\% CL Lower Limits (EPS-HEP 2011)}

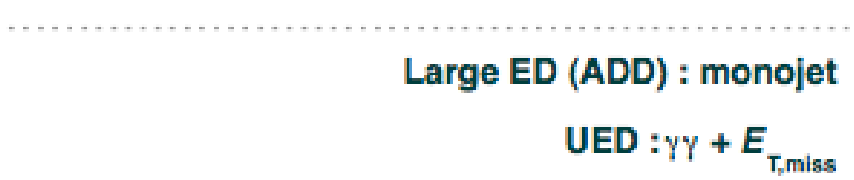

RS with $k / M_{\mathrm{Pl}}=0.1: m_{y}$ RS with $k / M_{\mathrm{PI}}=0.1: m_{\mathrm{eQ/yH}}$ RS with top couplings $g_{L}=1.0, g_{R}=4.0: m_{t}$ Quantum black hole (QBH) : $m_{\text {dijet }}, F(\chi)$

QBH : High-mass $\sigma_{t+x}$ ADD BH $\left(M_{\mathrm{th}} / M_{\mathrm{D}}=3\right):$ multijet $\Sigma p_{\mathrm{T}}, N_{\text {lets }}$ ADD BH $\left(M_{\mathrm{th}} / M_{\mathrm{D}}=3\right)$ : SS dimuon $N_{\text {ch. part. }}$ qqqq contact interaction : $F_{x}\left(m_{\text {dillet }}\right)$

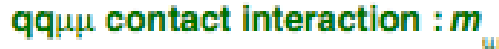

Scalar LQ pairs $(\beta=1)$ : kin. vars, in eejj, evj] Scalar LQ pairs $(\beta=1)$ : kin. vars. in $\mu \mu \mathrm{j}], \mu v \mathrm{j}]$ $4^{\text {th }}$ family : coll. mass in $Q_{4} \bar{Q}_{4} \rightarrow W q W q$ $4^{\text {th }}$ family : $\mathrm{d}_{4} \overline{\mathrm{d}}_{4} \rightarrow$ WtWt (SS dilepton) Major. neutr. $\left(V_{\text {4-ferm, }}, \Lambda=1 \mathrm{TeV}\right)$ : SS dilepton Excited quarks : $\boldsymbol{m}_{\text {dijet }}$ Axigluons : $m_{\text {diljet }}$ Color octet scalar : $\boldsymbol{m}_{\text {dilot }}$

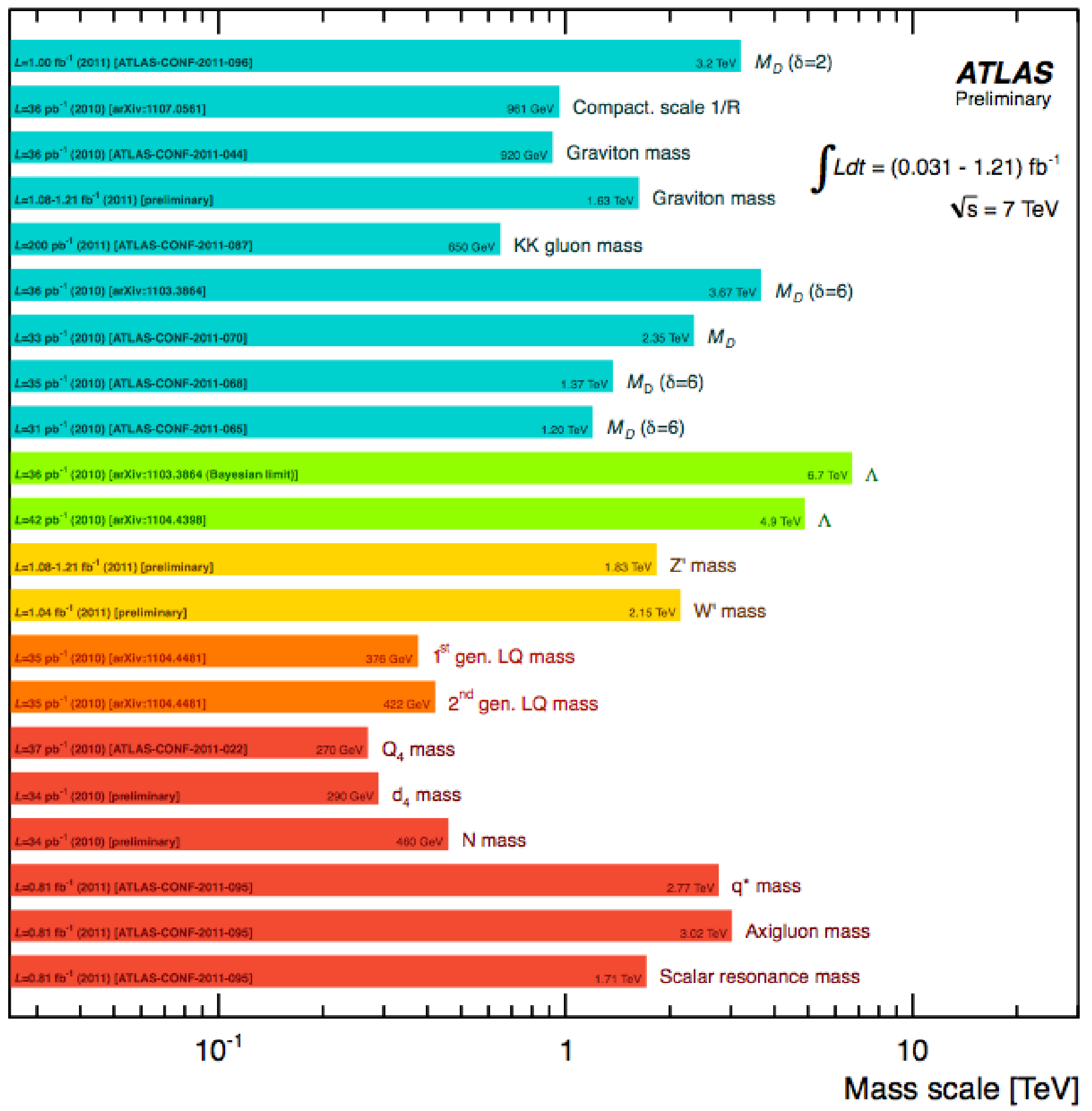




\section{Backup slides}




\section{A Toroidal LHC ApparatuS}
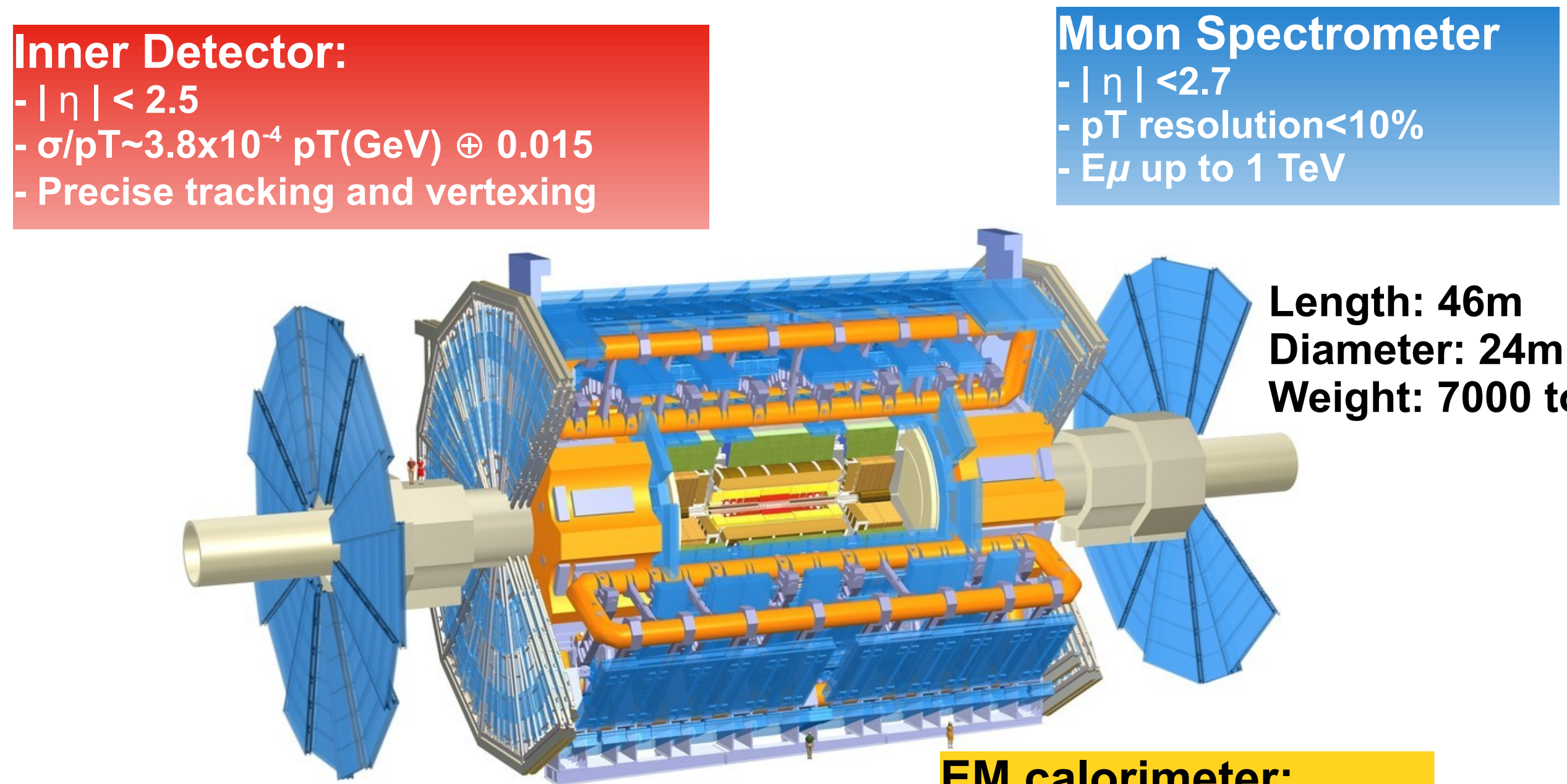

\section{HAD calorimeter}

$-|\eta|<5$

- Central: $\sigma / E \sim 50 \% / \sqrt{ } E \oplus 0.03$

- Forward: $\sigma / E \sim 90 \% / \sqrt{ } E \oplus 0.07$
Length: $46 \mathrm{~m}$ Diameter: $24 \mathrm{~m}$ Weight: 7000 tons
EM calorimeter:

$-|\eta|<3.2$

- Resolution $\sigma / E \sim 10 \% / \sqrt{ } E$ 


\section{LHC and ATLAS Operations}

> Good performance in 2010

(45 $\mathrm{pb}^{-1}$ recorded)

- Remarkable increase of LHC performance in $2011 ; 1.3 \mathrm{fb}^{-1}$ delivered up to now

- Good data taking efficiency in ATLAS

> New results with $\sim 1 \mathrm{fb}-1$

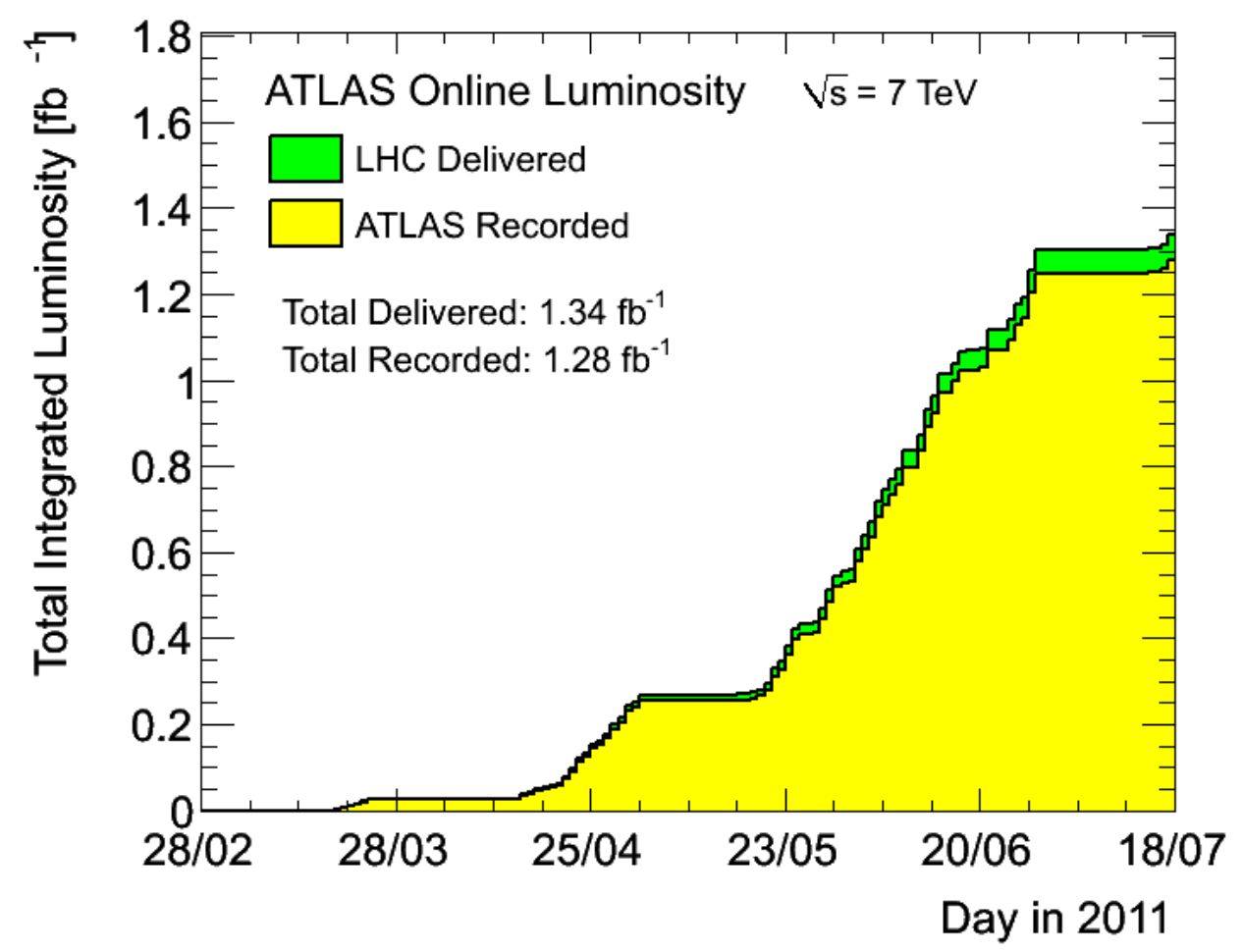

\begin{tabular}{|c|c|c|c|c|c|c|c|c|c|c|c|c|}
\hline \multicolumn{3}{|c|}{$\begin{array}{l}\text { Inner Tracking } \\
\text { Detectors }\end{array}$} & \multicolumn{4}{|c|}{ Calorimeters } & \multicolumn{4}{|c|}{ Muon Detectors } & \multicolumn{2}{|c|}{ Magnets } \\
\hline Pixel & SCT & TRT & $\begin{array}{l}\text { LAr } \\
\text { EM }\end{array}$ & $\begin{array}{l}\text { LAr } \\
\text { HAD }\end{array}$ & $\begin{array}{l}\text { LAr } \\
\text { FWD }\end{array}$ & Tile & MDT & $\mathrm{RPC}$ & $\mathrm{CSC}$ & TGC & Solenoid & Toroid \\
\hline 99.9 & 99.8 & 100 & 89.0 & 92.4 & 94.2 & 99.7 & 99.8 & 99.7 & 99.8 & 99.7 & 99.3 & 99.0 \\
\hline
\end{tabular}




\section{Diphoton with Large Missing Et}

Physics Model:

GMSB with $\widetilde{G} \operatorname{LSP}\left(\tilde{X}_{1} \rightarrow \gamma \widetilde{\mathrm{G}}\right)$

$\operatorname{UED}\left(\gamma^{*} \rightarrow \gamma+\mathrm{G}\right)$

> Observable

> Diphoton invariant mass

> Event Selection

> Two photons, Et>30(20) GeV

> missing Et>125 GeV

> Background

- fake photons from QCD, $\gamma+$ jet

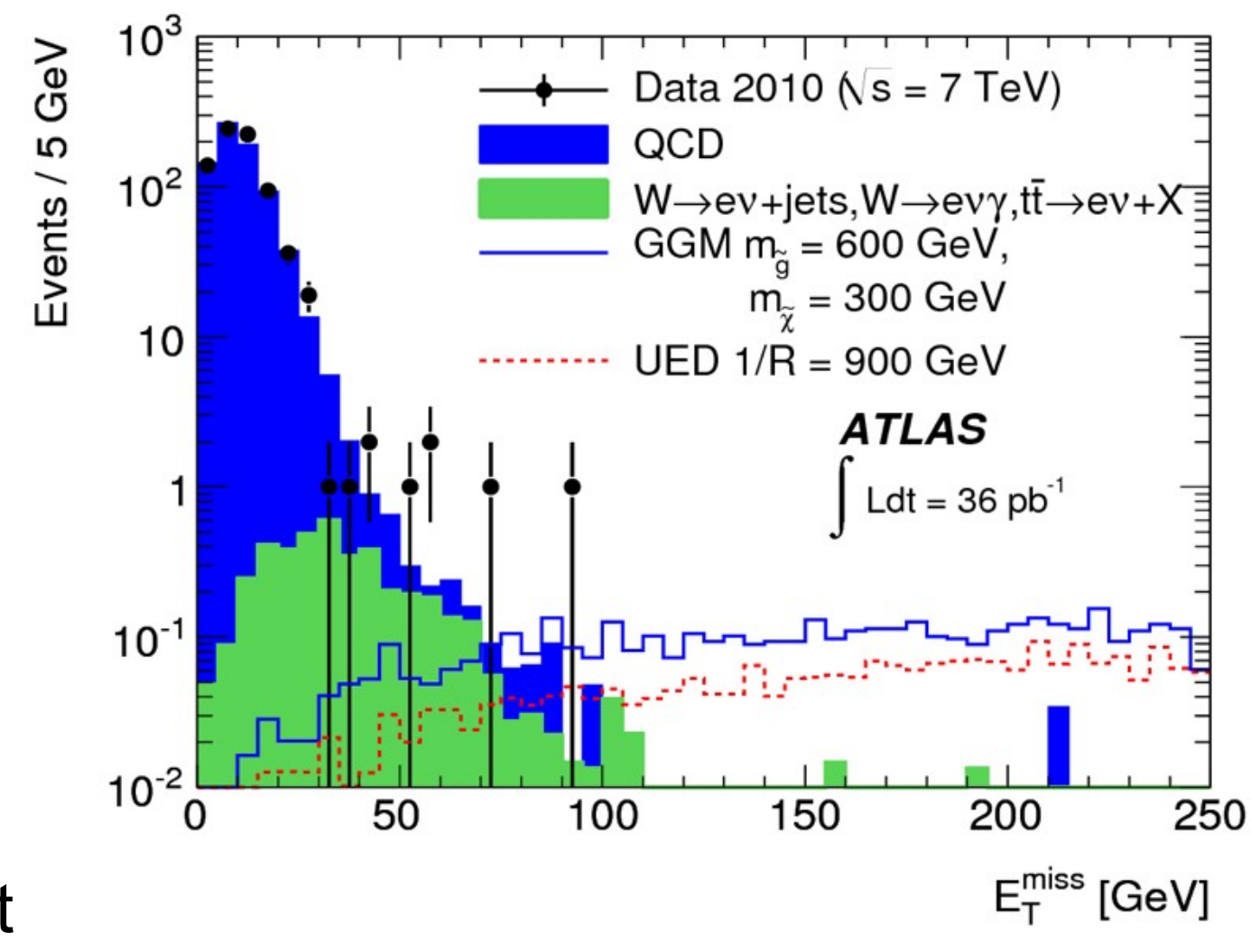

> $\mathrm{W}+\mathrm{X}$ and ttbar (electron and jet faking photons)

> No candidate events survive the selection 


\section{Dijet+lepton+MET final states}

Physics Models:

??

- Observable

- Dijet invariant mass

> Event Selection

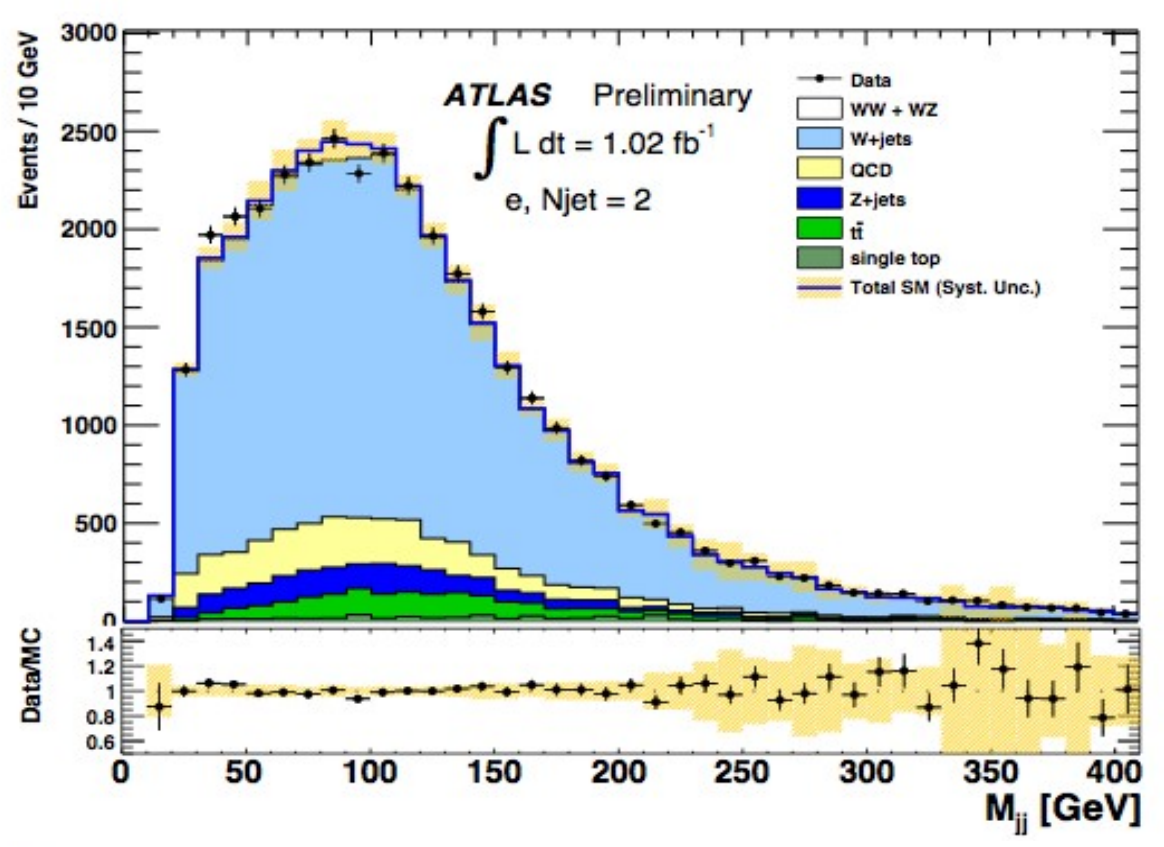

> Two jets with one lepton $(e, \mu)$ and MET

> Main backgrounds

> EW background (W+jets)

> Diboson (WW,WZ)

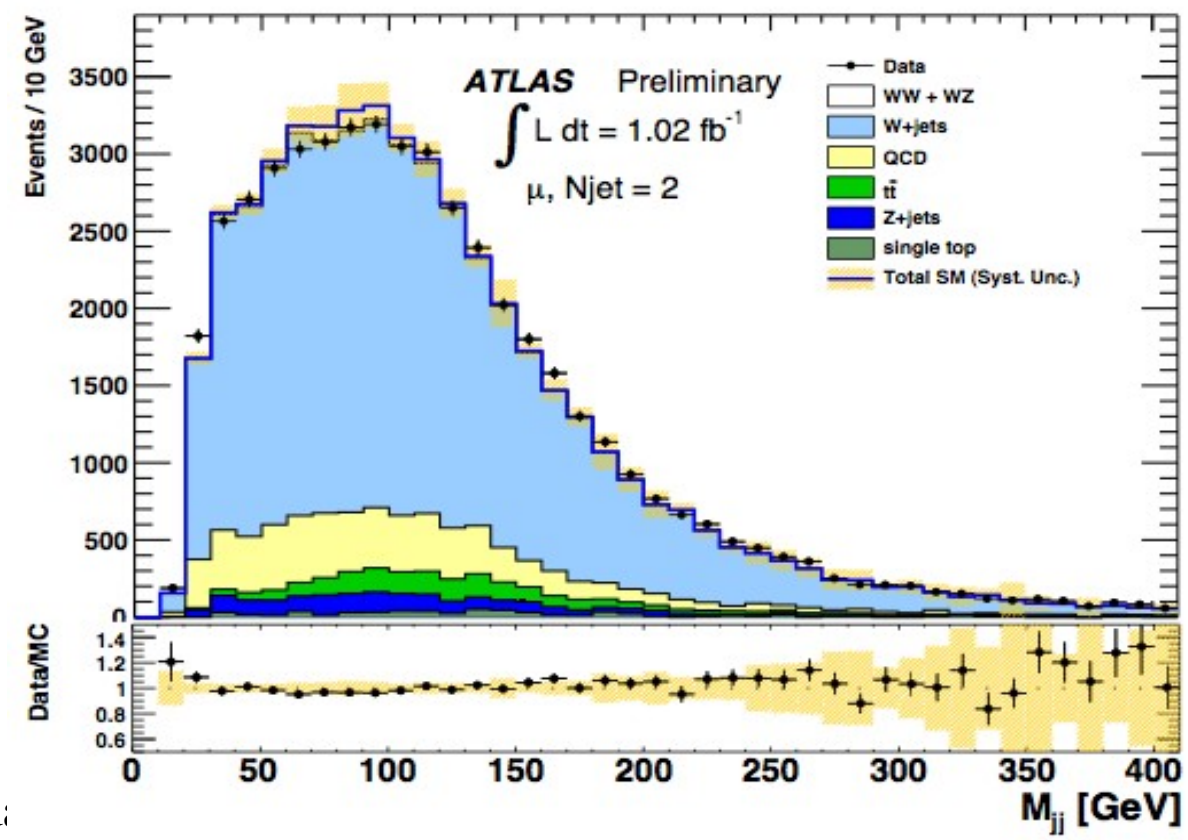




\section{ATLAS-CONF-2011-097 Dijet+lepton+MET final states}
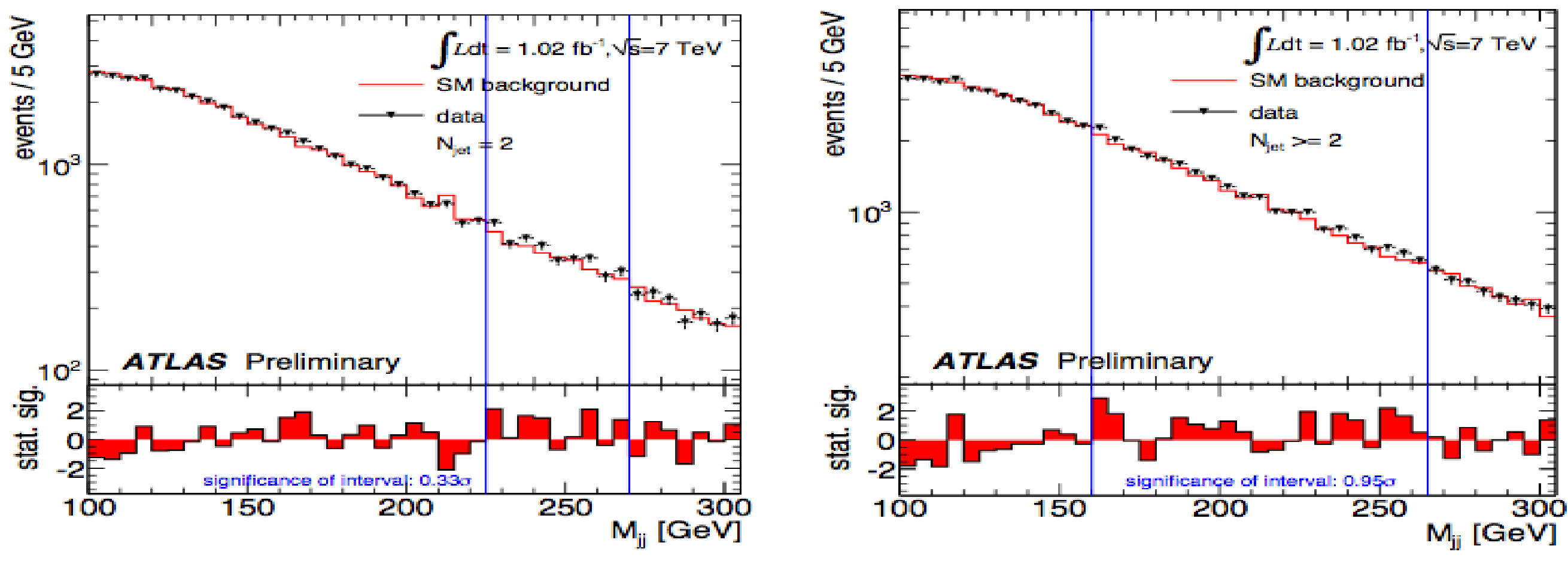

\section{No significant excess beyond the SM expectation is observed}




\section{Extra Spatial Dimensions}

- Alternative to solve the hierarchy problem

- Extra spatial dimensions explain the apparent weakness of Gravity (relevant scale $\sim 1 \mathrm{TeV}$ )
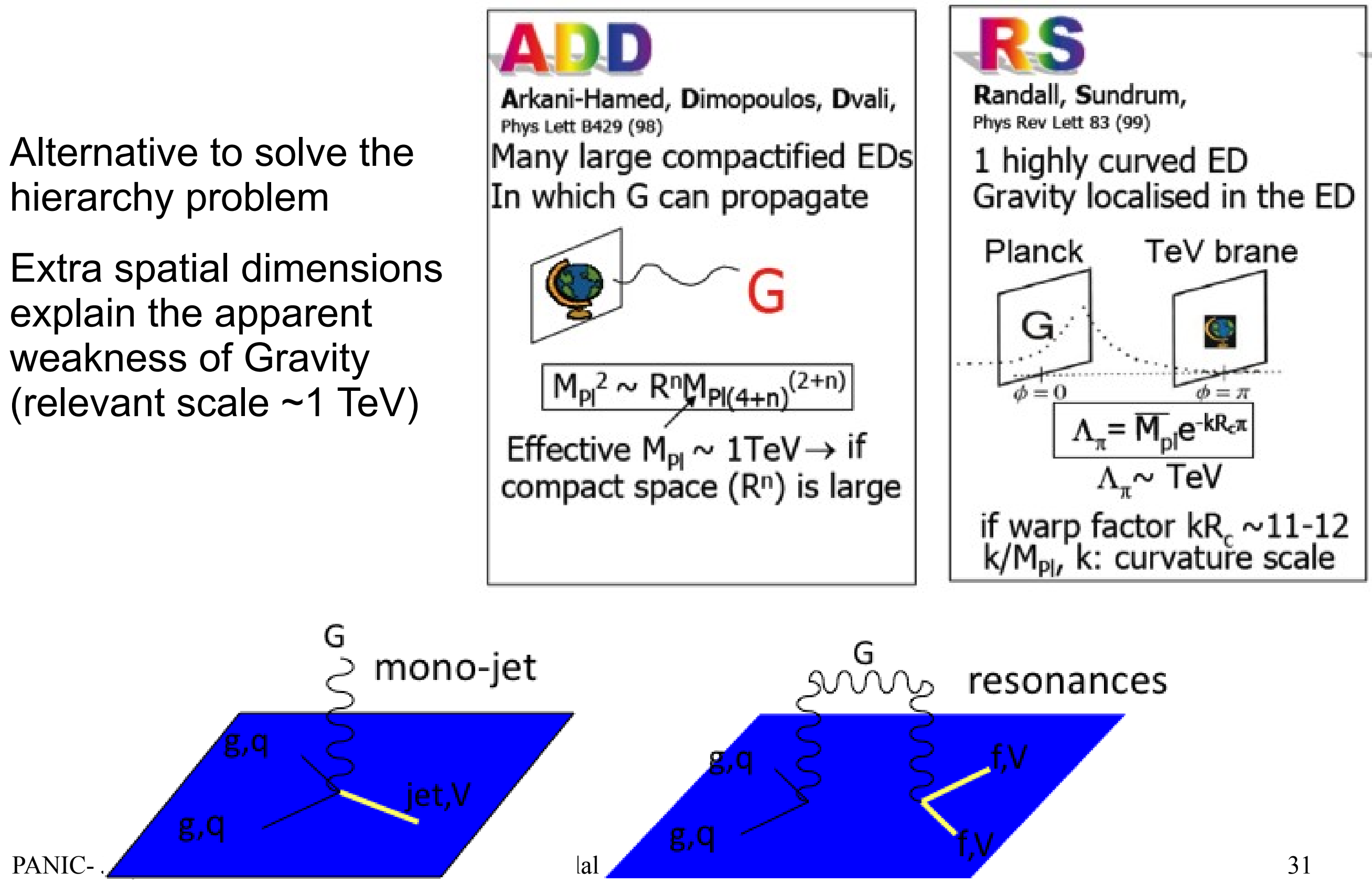